\title{
SINGULARITIES PRODUCED IN CONORMAL WAVE INTERACTIONS
}

\author{
LINDA M. HOLT
}

\begin{abstract}
Three problems on the interactions of conormal waves are considered. Two are examples which demonstrate that nonlinear spreading of singularities can occur when the waves are conormal. In one case, two of the waves are tangential, and the other wave is transversal to the first two. The third result is a noninteraction theorem. It is shown that under certain conditions, no nonlinear spreading of the singularities will occur.
\end{abstract}

\section{INTRODUCTION}

In the first problem, we consider the initial value problem in two space dimensions,

$$
\square u=\beta u^{3},\left.\quad u\right|_{t=0}=\left.v\right|_{t=0},\left.\quad u_{t}\right|_{t=0}=\left.v_{t}\right|_{t=0} .
$$

(Here $\left.\square=\partial_{t}^{2}-\partial_{x}^{2}-\partial_{y}^{2}.\right) \quad v \in H^{s}\left(\mathbf{R}^{3}\right)$ satisfies $\square v=0$ and is given by $v=$ $v_{1}+v_{2}+v_{3}$, where each $v_{i}$ has a restricted type of singularity at $t=0:$ namely, $\left.v_{1}\right|_{t=0}$ and $\left.v_{2}\right|_{t=0}$ are both conormal with respect to $\{y=0, x=y=0\}$, with wave front set over the origin a single ray (in opposite directions), and $\left.v_{3}\right|_{t=0}$ is conormal with respect to $\{x=0\}$, with its wave front set over the origin being a single ray. (A distribution $w$ is conormal with respect to such smoothly embedded submanifolds $S_{1}, S_{2}, \ldots, S_{k}$ if $N_{1} \cdots N_{j} w \in H_{\text {loc }}^{s}\left(\mathbf{R}^{3}\right)$ for all smooth vector fields $N_{1}, \ldots, N_{j}$, each of which is tangent to all of $S_{1}, S_{2}, \ldots, S_{k}$.) The aim is to show that if the initial data has this type of conormal regularity, then a spreading of singularities can occur, even if $v_{3}=0$. These new singularities have strength roughly $H^{3 s}$. If instead of having two functions conormal to $\{y=0, x=y=0\}$, and one conormal to $\{x=0\}$, the situation is reversed (i.e. one and two, respectively), there is no spreading of singularities. Together, these results clarify the roles of such conormal singularities in nonlinear interaction.

It has been shown previously that if $u$ satisfies

$$
\left(\partial_{x} \partial_{y}+\partial_{x} \partial_{z}+\partial_{y} \partial_{z}\right) u=\beta u^{3}, \quad u=x_{+}^{k}+y_{+}^{k}+z_{+}^{k}
$$

for $x+y+z<-2, k$ an integer (so we have singularities on three transversal planes) then new singularities arise on the surface of the light cone over the origin with strength roughly $H^{3 k}$. In the second problem, we examine a similar situation, except that two of the characteristic hypersurfaces are tangential and

Received by the editors July 20, 1992 and, in revised form, March 11, 1994.

1991 Mathematics Subject Classification. Primary 35L05, 35L70.

(C)1995 American Mathematical Society 
the other is transversal to the other two. This is the situation which would arise if, at a later time, another plane wave intersected the line of tangency of the light cone and one of the original plane waves in the above problem. We show, for a solution to the following semilinear system:

$$
\begin{aligned}
& \tilde{\square}\left(\begin{array}{l}
u_{1} \\
u_{2} \\
u_{3} \\
u_{4}
\end{array}\right)=\left(\begin{array}{c}
0 \\
0 \\
0 \\
\beta u_{1} u_{2} u_{3}
\end{array}\right), \\
& \left(\begin{array}{l}
u_{1} \\
u_{2} \\
u_{3} \\
u_{4}
\end{array}\right)_{\{t<0\}}=\left(\begin{array}{c}
v_{1} \\
v_{2} \\
v_{3} \\
0
\end{array}\right)_{\{t<0\}},
\end{aligned}
$$

(here $\tilde{\square}$ is defined by $\left.\tilde{\square}=\partial_{y} \partial_{z}-\partial_{x}^{2}+y \partial_{y}^{2}-(m-1) \partial_{y}\right)$ that $u_{4}$ has new singularities on the surface of the light cone, with smoothness approximately $H^{5 m}$ microlocally away from the original singularities, where $v_{i} \in H^{m}$.

The research in this paper was conducted for my doctoral dissertation at Rutgers University. I would like to thank my advisor, Professor Michael Beals, for his generous help and encouragement.

\section{Preliminaries}

Our aim is to show that, for solutions to $\square u=\beta u^{3}$, where $\square$ is the usual wave operator in two space dimensions $\left(\square=\partial_{t}^{2}-\partial_{x}^{2}-\partial_{y}^{2}\right)$, a spreading of singularities compared to the linear case can occur for initial data of the conormal type described above. We first recall some definitions and preliminary results; see for example Chazarain-Piriou [7], Nirenberg [11], and Beals [4].

Theorem 2.1 (Linear Energy Estimate). Let $p(x, D)$ be a partial differential operator of order $m$ on $\mathbf{R}^{n+1}$, with coefficients of at most linear growth, strictly hyperbolic with respect to the planes $\left\{x_{n+1}=c\right\}$, and let $u$ satisfy $p(x, D) u$ $=f(x)$. Assume that $\left\{x_{n+1}=c\right\}$ are uniformly noncharacteristic. If $f \in$ $H_{\mathrm{loc}}^{s-m+1}\left(\mathbf{R}^{n+1}\right)$ and $u \in H_{\mathrm{loc}}^{s}\left(x:\left|x_{n+1}\right|<\varepsilon\right)$ for some $\varepsilon>0$, then $u \in$ $H_{\mathrm{loc}}^{s}\left(\mathbf{R}^{n+1}\right)$.

Recall, we say $u \in H_{m l}^{s}\left(x_{0}, \xi_{0}\right)$ if there exists $\varphi \in C_{0}^{\infty}\left(\mathbf{R}^{n}\right), \varphi\left(x_{0}\right) \neq 0$, and a conic neighborhood $\Gamma \subset \mathbf{R}^{n}$ of $\xi_{0}$ such that $\langle\xi\rangle^{s} \chi_{\Gamma}(\xi)(\widehat{\varphi u})(\xi) \in L^{2}\left(\mathbf{R}^{n}\right)$.

Under appropriate regularity conditions, the microlocal singularities of $u$ will propagate along the null bicharacteristics.

Theorem 2.2 (Hörmander). Let $p(x, D)$ be a strictly hyperbolic partial differential operator of order $m$ on $\mathbf{R}^{n+1}$, let $p_{m}\left(x_{0}, \xi_{0}\right)=0$, and let $\Gamma$ be the null bicharacteristic through $\left(x_{0}, \xi_{0}\right)$ for $p_{m}$. If $p(x, D) u=f(x), f \in H_{m l}^{s-m+1}(\Gamma)$, and $u \in H_{m l}^{s}\left(x_{0}, \xi_{0}\right)$, then $u \in H_{m l}^{s}(\Gamma)$.

For nonlinear problems, Schauder's Lemma describes the action of smooth functions $f$ on the Sobolev spaces: If $u \in H_{\text {loc }}^{s}\left(\mathbf{R}^{n}\right), s>n / 2$, then $f(u) \in$ $H_{\text {loc }}^{s}\left(\mathbf{R}^{n}\right)$. A microlocal analogue is the following:

Lemma 2.3 (Rauch). Let $u_{1}, u_{2} \in H^{s}\left(\mathbf{R}^{n}\right)$ for $s>n / 2$, and suppose that $\Pi_{\xi} W F\left(u_{i}\right) \subset K_{i}$ for $i=1,2$. If $K_{3} \subset\left(K_{1} \cup K_{2}\right)^{c}$, then for any $\delta>0$, $u_{1} u_{2} \in H_{m l}^{2 s-n / 2-\delta}\left(\mathbf{R}^{n} \times K_{3}\right)$. 

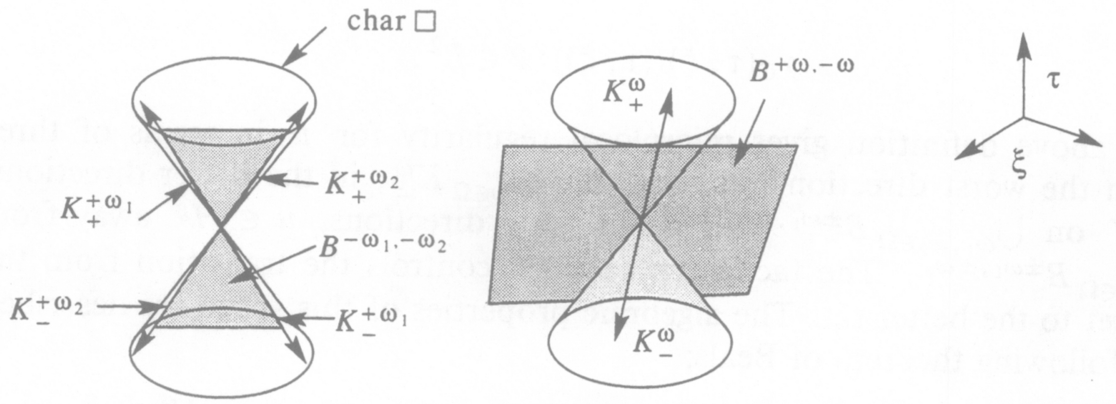

FIGURE 1. Illustration for $H^{s, r, g}\left(\mathbf{R}^{n}\right)$

In this work, we consider singularities of a restricted type, called conormal.

Definition 2.4. Let $S_{1}, \ldots, S_{k}$ be a family of smoothly embedded submanifolds of $\mathbf{R}^{n}$, with pairwise intersections which are also smoothly embedded. A distribution $u \in H^{s}\left(\mathbf{R}^{n}\right)$ is said to be conormal with respect to $S_{1}, S_{2}, \ldots, S_{k}$ if $N_{1} N_{2} \cdots N_{j} u \in H^{s}\left(\mathbf{R}^{n}\right)$ for all $N_{1}, \ldots, N_{j}$ vector fields which are simultaneously tangent to all of $S_{1}, \ldots, S_{k}$.

In the case of smooth hypersurfaces with transverse intersections, this definition is standard. For more complicated intersections (and nonsmooth manifolds) different notions of conormal are also useful; see for example MelroseRitter [10] and Sá Barreto [16].

In order to measure strength of singularities in certain directions, we use the microlocal algebra defined by $M$. Beals in [1]. Note that we assume that all distributions considered have been multiplied by smooth cutoff functions so as to be compactly supported, and that all results are considered to be local. (See Figure 1.)

Definition 2.5. Let $\Omega \subset S^{n-1}$ be a closed subset (possibly empty), and for $\omega \in \Omega$, let $K_{ \pm}^{\omega}$ be the rays through $\pm(1, \omega)$ in $\mathbf{R}^{n+1} \backslash 0$. Set $B^{+\omega,+\omega}=$ $K_{+}^{\omega}, B^{-\omega,-\omega}=K_{-}^{\omega}$,

$$
B^{+\omega,-\omega}=B^{\omega}=\{\text { tangent plane to char } \square \text { at } \pm(1, \omega)\} \text {. }
$$

For $\omega_{1} \neq \omega_{2}, \omega_{1}, \omega_{2} \in \Omega$, set $B^{ \pm \omega_{1}, \pm \omega_{2}}=\operatorname{closure}\left(K_{ \pm}^{\omega_{1}}+K_{ \pm}^{\omega_{2}}\right)$.

Definition 2.6. For $\Omega \subset S^{n-1}$ closed and $s \leq r \leq g, H_{\Omega}^{s, r, g}$ consists of those distributions $u$ satisfying

(1) If $K_{ \pm}$is a small conic neighborhood of $\pm(1, \omega), \omega \in \Omega$, then

$$
\chi_{ \pm K}(\tau, \xi)\langle(\tau, \xi)\rangle^{s}\langle\tau \mp|\xi|\rangle^{r-s} \hat{u} \in L^{2} .
$$

(2) If $B$ is a small conic neighborhood of

$$
\left(\tau_{0}, \xi_{0}\right) \in \bigcup_{\omega_{1}, \omega_{2} \in \Omega} B^{ \pm \omega_{1}, \pm \omega_{2}} \backslash \bigcup_{\omega \in \Omega} K_{ \pm}^{\omega},
$$

then

$$
\chi_{B}(\tau, \xi)\langle(\tau, \xi)\rangle^{r} \hat{u} \in L^{2} .
$$

(3) If $G$ is a small conic neighborhood of

$$
\left(\tau_{0}, \xi_{0}\right) \in \mathbf{R}^{n+1} \backslash \bigcup_{\omega_{1}, \omega_{2} \in \Omega} B^{ \pm \omega_{1}, \pm \omega_{2}},
$$


then

$$
\chi_{G}(\tau, \xi)\langle(\tau, \xi)\rangle^{g} \hat{u} \in L^{2}
$$

The above definition gives microlocal regularity for $u$ in terms of three sets: In the worst directions, $u \in H^{s}$ on $\bigcup_{\omega \in \Omega} K_{ \pm}^{\omega}$; in the better directions, $u \in H^{r}$ on $\bigcup_{\omega_{1}, \omega_{2} \in \Omega} B^{ \pm \omega_{1}, \pm \omega_{2}}$; in the good directions, $u \in H^{g}$ away from $\bigcup_{\omega_{1}, \omega_{2} \in \Omega} B^{ \pm \omega_{1}, \pm \omega_{2}}$. The factor $\langle\tau \mp|\xi|\rangle^{r-s}$ controls the transition from the worst set to the better set. The algebraic properties of this space are described in the following theorem of Beals:

Theorem 2.7. Let $n / 2<s_{1} \leq s_{2}, s_{i}+1 / 2 \leq r_{i} \leq g_{i}$. If $u_{i} \in H_{\Omega}^{s_{i}, r_{i}, g_{i}}$, then $u_{1} u_{2} \in H_{\Omega}^{s_{1}, \rho, \gamma}$ where $\rho=\min \left(r_{1}, r_{2}+s_{1}-s_{2}, s_{1}+s_{2}-n / 2-\varepsilon\right)$ and $\gamma=$ $\min \left(g_{1}, g_{2}, s_{1}+r_{2}-n / 2-\varepsilon, s_{2}+r_{1}-n / 2-\varepsilon\right)$. In particular, $H^{s, r, g}$ is an algebra for $n / 2<s, s+1 / 2 \leq r \leq g, r<2 s-n / 2, g<s+r-n / 2$.

The following result concerns the interaction of the forward fundamental solution $E$ for $\square$ and the spaces $H^{s, r, g}(\square E w=w, E w=\partial / \partial t E w=0$ for $t=0)$. In general, $E$ improves overall Sobolev regularity by one. However, if we examine the result microlocally, we find that in the elliptic directions the regularity is improved by two, and the transition between the characteristic and elliptic directions is respected as in (1) of Definition 2.6.

Lemma 2.8. Let $w \in H_{\Omega}^{s, r, g}, s \leq r$, and let $E$ be the forward fundamental solution for $\square$. Then $E w \in H_{\Omega}^{s+1, r+2, g+1}$.

These algebra and propagation results are shown in [1] to lead to solutions of $\square u=f(u), f$ smooth, $u$ and $(\partial / \partial t) u$ at time zero singular only at the origin, such that $u$ is singular on the solid light cone over the origin. (In the linear case, the singularities would only be present on the surface of the light cone.)

\section{DEFINITIONS}

The following problem will be considered:

$$
\square u=\beta u^{3},\left.\quad u\right|_{t=0}=\left.v\right|_{t=0},\left.\quad u_{t}\right|_{t=0}=\left.v_{t}\right|_{t=0} .
$$

Here $v$ is a solution to the linear wave equation $\square v=0$, and at $t=0, v$ is a sum of functions, $v=\tilde{u}_{+}+\tilde{u}_{-}+v_{+}$, the first two being conormal with respect to $\{y=0, x=y=0\}$ and having wave front set over the origin a single ray (in opposite directions), and the other conormal with respect to $\{x=0\}$ with its wave front set over the origin being a single ray. The aim is to show that if the initial data has this type of conormal regularity, then spreading of singularities can occur. (In the linear case, the subsequent wave front would contain three null bicharacteristics with $(t, x, y)$-projections onto two lines through the origin.)

Define $\tilde{u}_{+}, \tilde{u}_{-}$, and $v_{+}$by their partial Fourier Transforms, as follows. Note that $d \xi$, where here $\xi=\left(\xi_{1}, \xi_{2}\right)$ represents the dual variables, is normalized to $(2 \pi)^{-n}$ times the usual Lebesgue measure.

$$
\tilde{u}_{+}=e^{i t|\xi|} \frac{\varphi\left(\frac{\left|\xi_{1}\right|^{1+\varepsilon}}{\xi_{2}}\right) \gamma\left(\xi_{2}\right)}{\left\langle\xi_{2}\right\rangle^{s+1+\varepsilon}}=e^{i t|\xi|} \hat{f}(\xi)
$$


where

$$
\begin{gathered}
\hat{f}(\xi)=\frac{\varphi\left(\frac{\left|\xi_{1}\right|^{1+\varepsilon}}{\xi_{2}}\right) \gamma\left(\xi_{2}\right)}{\left\langle\xi_{2}\right\rangle^{s+1+\varepsilon}}, \quad \tilde{u}_{-}=e^{-i t|\xi|} \hat{f}(-\xi) \\
v_{+}=e^{i t|\xi|} \frac{\varphi\left(\xi_{2}\right) \gamma\left(\xi_{1}\right)}{\left\langle\xi_{1}\right\rangle^{s+1 / 2+\varepsilon}}=e^{i t|\xi|} \hat{g}(\xi),
\end{gathered}
$$

where

$$
\hat{g}(\xi)=\frac{\varphi\left(\xi_{2}\right) \gamma\left(\xi_{1}\right)}{\left\langle\xi_{1}\right\rangle^{s+1 / 2+\varepsilon}} .
$$

Here $\varepsilon>0$ is fixed, $\gamma$ is a $C^{\infty}$ function, supported in $\{x>1 / 2\}$ and 1 for $x>1 . \varphi$ and $\beta$ are $C_{0}^{\infty}$ functions, supported in a neighborhood of 0 , and $\hat{f}(\xi)$ is supported in $\left\{\xi_{2}>1\right\}$. Then

$$
-1<\frac{\left|\xi_{1}\right|^{1+\varepsilon}}{\xi_{2}}<1
$$

on $\operatorname{supp} \hat{f}(\xi)$, while $\hat{g}(\xi)$ is supported in $\left\{\xi_{1}>1\right\}$, and $\left|\xi_{2}\right|<1$ on $\operatorname{supp} \hat{g}(\xi)$.

Notation. The following notation will be used: $a \approx b$ means $a \leq c_{1} b$ and $b \leq c_{2} a$ for some constants $c_{1}>0, c_{2}>0$. $a \lesssim b$ means $a \leq c_{3} b$ for some constant $c_{3}>0$. The symbol $\gtrsim$ is defined similarly.

Note that $f$ is conormal in the strong sense to the pair of submanifolds consisting of the line $\{y=0\}$ and the point $\{(0,0)\}$, i.e., it is infinitely differentiable with respect to $y \partial_{y}, x \partial_{x}, y \partial_{x}$. These are the vector fields simultaneously tangent to $\{y=0\}$ and the point $\{x=0, y=0\}$. This fact is easily verified by checking the action of $\xi \partial_{\xi}, \eta \partial_{\eta}$, and $\xi \partial_{\eta}$ on $\hat{f}$.

Also note that $g$ is clearly conormal with respect to the line $x=0$. It follows immediately that the wave front set $\{(t, x, y ; \tau, \xi, \eta)\}$ of $v$ passing over the origin $(0,0,0)$ is $\{(r, 0,-r ; \tau, 0, \tau): \tau>0\} \cup\{(r, 0,-r ;-\tau, 0,-\tau): \tau>$ $0\} \cup\{(r,-r, 0 ; \tau, \tau, 0): \tau>0\}$.

Theorem 3.1. Let $s>3 / 2, \beta=\alpha^{2} \in C_{0}^{\infty}(\mathbf{R})$ with support sufficiently near to $0, \hat{\beta} \geq 0, \hat{\beta}(0)>0$, and let $u \in H^{s}\left(\mathbf{R}^{n}\right)$ be the solution of $\square u=\beta u^{3},\left.u\right|_{t=0}=$ $\left.v\right|_{t=0},\left.u_{t}\right|_{t=0}=\left.v_{t}\right|_{t=0}$ with $v$ as above. Then $u$ has new singularities on the surface of the light cone which are not present in $v$. In particular, we have $u \in H_{m l}^{3 s-1 / 2-\varepsilon^{\prime \prime}}(r, r, 0 ; \tau,-\tau, 0), r>0, \varepsilon^{\prime \prime} \rightarrow 0$ as $\varepsilon \rightarrow 0$, but $u \notin$ $H_{m l}^{3 s-1 / 2-\varepsilon^{\prime \prime}+\delta}(r, r, 0 ; \tau,-\tau, 0)$ for any $\delta>0$. (In the linear case, Hörmander's Theorem would yield $u \in H_{m l}^{\infty}(r, r, 0 ; \tau,-\tau, 0)$.)

The proof of this theorem is contained in $\$ \S 4-10$.

In Beals [1], in the general (not conormal) case, it was shown that for $H^{s}$ solutions to the semilinear wave equation, there is a choice of initial data such that a nonlinear singularity is produced of order $H^{3 s+\varepsilon}$. In a related problem, Bony [5] and Melrose-Ritter [10], showed that for solutions conormal in the past of strength $H^{s}$ with respect to three characteristic surfaces, the solution is conormal in the future with respect to the original surfaces and the light cone over the point of triple intersection. The solution has microlocal regularity of 
at least $H^{2 s-n / 2+1}$ on the light cone, where $n$ is the number of dimensions. An analogous result is proved for the Cauchy problem in [6].

\section{LOWER BOUNDS FOR DOUBLE INTERACTION}

We will be analyzing in detail two terms of $v^{3}$, with $v$ as in Theorem 3.1: $\tilde{u}_{+} \tilde{u}_{-} v_{+}$and $\tilde{u}_{+}^{2} \tilde{u}_{-}$. In particular, we will be finding both lower and upper bounds for these terms. We will begin by finding lower bounds for $\tilde{u}_{+} v_{+}$, in order to analyze the $\tilde{u}_{+} \tilde{u}_{-} v_{+}$term.

$$
\begin{aligned}
\alpha\left(\widehat{t) \hat{u}_{+}} v_{+}\left(\tau, \xi_{1}, \xi_{2}\right)\right. & =\int e^{-i \tau} \alpha(t) \int \tilde{u}_{+}^{-}(\xi-\eta) v_{+}^{-}(\eta) d \eta d t \\
& =\int \hat{\alpha}(\tau-|\xi-\eta|-|\eta|) \hat{f}(\xi-\eta) \hat{g}(\eta) d \eta
\end{aligned}
$$

(We can assume without loss of generality that $\hat{\alpha}(\tau) \geq 0$.) By the rapid decrease of $\hat{\alpha}$, we can assume that $|\tau-| \xi-\eta|-| \eta|| \lesssim|\xi|^{\delta}$ for small $\delta>0$.

In the integrand, $\hat{\alpha}$ is supported where

$$
\tau-|\xi|^{\delta}<|\xi-\eta|+|\eta|<\tau+|\xi|^{\delta} .
$$

This is a thickened ellipse in the $\left(\eta_{1}, \eta_{2}\right)$ plane, with foci at 0 and $\xi$, for fixed $\tau$. See Figure 2 .

For large $\tau$, and $|\xi|<(1-\varepsilon) \tau,(|\xi|, \tau>0)$, the thickness of the ellipse is $\approx|\xi|^{\delta}$.

With $f$ and $g$ defined as in $\S 3$, we have

$$
\hat{f}(\xi-\eta)=\frac{1}{\left\langle\xi_{2}-\eta_{2}\right\rangle^{s+1+\varepsilon}}
$$

for $-(1 / 2)\left(\xi_{2}-\eta_{2}\right)<\left(\xi_{1}-\eta_{1}\right)^{1+\varepsilon}<(1 / 2)\left(\xi_{2}-\eta_{2}\right), \xi_{2}-\eta_{2} \geq 1$, and

$$
\hat{g}(\eta)=\frac{1}{\left\langle\eta_{1}\right\rangle^{s+1 / 2+\varepsilon}}
$$

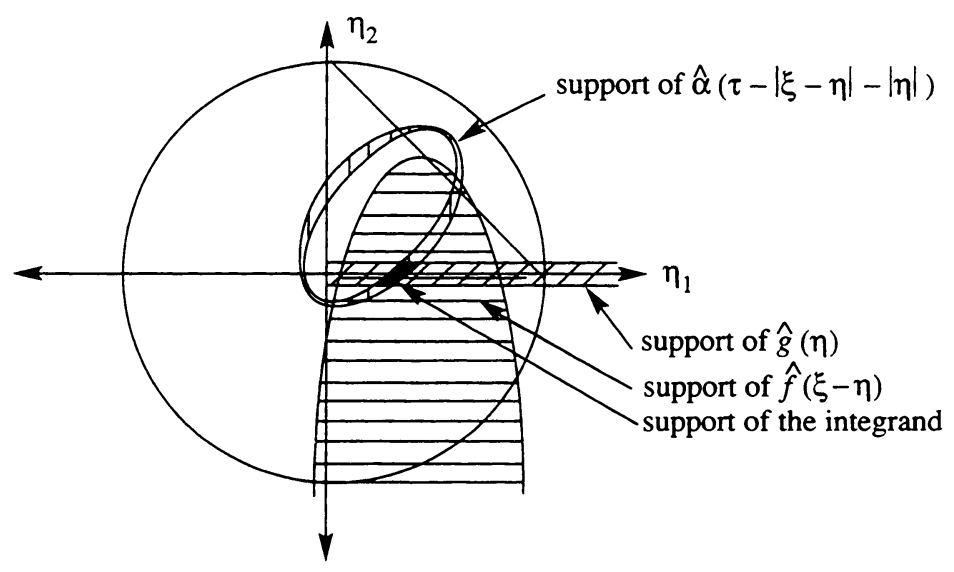

FiguRE 2. Regions of lower bounds 


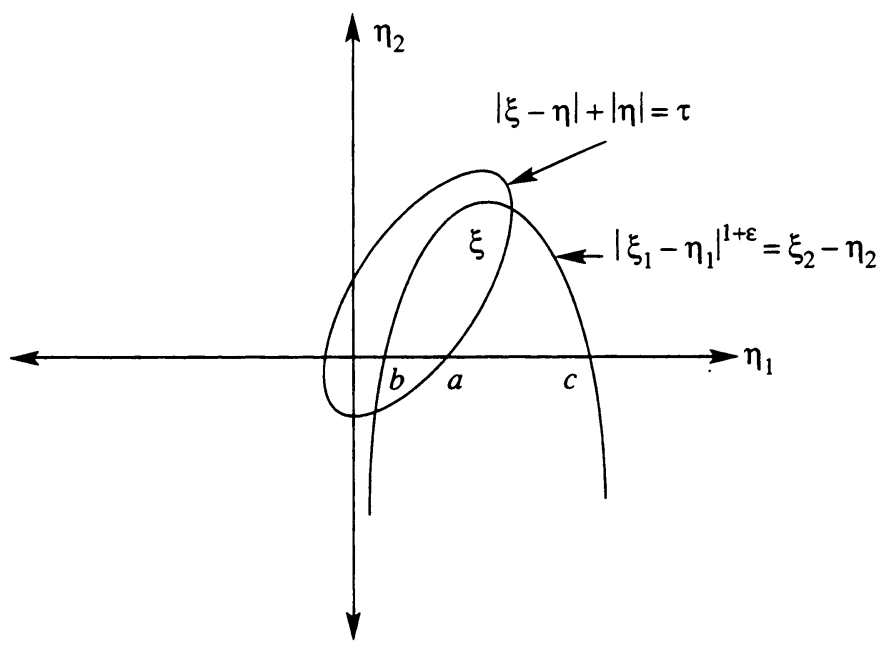

FIGURE 3. Intersection of ellipse with $\eta_{1}$ axis

for $\eta_{1} \geq c>1,-1<\eta_{2}<1$. Assume for simplicity that $\hat{\alpha}(\tau-|\xi-\eta|-|\eta|) \geq 1$ for $|\tau-| \xi-\eta|-| \eta||<1$.

The integral has bounds from below when the thickened ellipse, as well as the two regions above, intersect. In particular, it is bounded from below on the line segment connecting $\left(\xi_{1}, \xi_{2}\right)=(\tau, 0)$ to $(0, \tau)$, for fixed large $\tau$, and so we restrict our attention to this set.

Claim. We get a triple intersection of the above regions for sufficiently large $\tau$, for $\xi$ in a thin triangular wedge, with vertices:

$$
(\tau, 0), \quad\left((1 / 2) \tau^{1 /(1+\varepsilon)}, \tau\right), \quad\left(-(1 / 2) \tau^{1 /(1+\varepsilon)}, \tau\right) .
$$

In order that we get a triple intersection, it is sufficient to have that the positive $\eta_{1}$, where the ellipse $|\xi-\eta|+|\eta|=\tau$ meets the $\eta_{1}$ axis, lies between the two points where $\left|\xi_{1}-\eta_{1}\right|^{1+\varepsilon}=\xi_{2}-\eta_{2}$ meets the $\eta_{1}$ axis.

In other words (see Figure 3), it is sufficient to have $b \leq a \leq c$.

The ellipse intersects the positive $\eta_{1}$ axis at

$$
\eta_{1}=\frac{\tau^{2}-\xi_{1}^{2}-\xi_{2}^{2}}{2 \tau-2 \xi_{1}}=a
$$

The curve $\left|\xi_{1}-\eta_{1}\right|^{1+\varepsilon}=\xi_{2}-\eta_{2}$ intersects the $\eta_{1}$ axis at $b=\xi_{1}-\xi_{2}^{1 /(1+\varepsilon)}$, and at $c=\xi_{1}+\xi_{2}^{1 /(1+\varepsilon)}$.

If $\xi$ lies in the wedge defined above and $\xi_{2}=s \tau, 0 \leq s \leq 1$, then

$$
\tau-s\left(\tau+(1 / 2) \tau^{1 /(1+\varepsilon)}\right)<\xi_{1}<\tau-s\left(\tau-(1 / 2) \tau^{1 /(1+\varepsilon)}\right) .
$$

Claim. $b<a$ for sufficiently large $\tau$. 
Proof. We write

$$
\begin{aligned}
a-b= & \frac{\tau^{2}-\xi_{1}^{2}-\xi_{2}^{2}}{2 \tau-2 \xi_{1}}-\left(\xi_{1}-\xi_{2}^{1 /(1+\varepsilon)}\right) \\
= & \xi_{2}^{1 /(1+\varepsilon)}-\frac{\xi_{2}^{2}}{2\left(\tau-\xi_{1}\right)}+\tau / 2-\xi_{1} / 2 \\
\geq & (s \tau)^{1 /(1+\varepsilon)}-\frac{s^{2} \tau^{2}}{2 s\left(\tau-(1 / 2) \tau^{1 /(1+\varepsilon)}\right)} \\
& +\tau / 2-\left\{\tau / 2-(s / 2)\left(\tau-(1 / 2) \tau^{1 /(1+\varepsilon)}\right)\right\} \\
= & s^{1 /(1+\varepsilon)} \tau^{1 /(1+\varepsilon)}-(s / 2) \tau^{1 /(1+\varepsilon)}\left(\frac{1-(1 / 4) \tau^{-\varepsilon /(1+\varepsilon)}}{1-(1 / 2) \tau^{-\varepsilon /(1+\varepsilon)}}\right) .
\end{aligned}
$$

Since $s^{1 /(1+\varepsilon)}>s$ and the quotient involving $\tau^{-\varepsilon /(1+\varepsilon)}$ is near 1 for large $\tau$, it follows that $a-b>0$ for large $\tau$.

Similarly, $a<c$ for sufficiently large $\tau$.

Lower bound for $\alpha \widetilde{u}_{+} v_{+}$.

$$
\alpha{\widehat{u}+v_{+}}\left(\tau, \xi_{1}, \xi_{2}\right)=\int \hat{\alpha}(\tau-|\xi-\eta|-|\eta|) \hat{f}(\xi-\eta) \hat{g}(\eta) d \eta .
$$

For $\xi$ within the wedge described in the claim above,

$$
\begin{gathered}
\hat{\alpha}(\tau-|\xi-\eta|-|\eta|) \geq 1, \\
\hat{f}(\xi-\eta)=1 /\left\langle\xi_{2}-\eta_{2}\right\rangle^{s+1+\varepsilon} \gtrsim 1 /\langle\xi\rangle^{s+1+\varepsilon}
\end{gathered}
$$

since $\left|\xi_{2}-\eta_{2}\right| \lesssim|\xi|$, and

$$
\hat{g}(\eta)=1 /\left\langle\eta_{1}\right\rangle^{s+1 / 2+\varepsilon} \gtrsim 1 /\langle\xi\rangle^{s+1+\varepsilon}
$$

since $\eta_{1} \lesssim|\xi| . \quad\left(\eta_{1} \leq \tau \leq \sqrt{5}|\xi|\right)$. Set $R=\{\eta:-1 \leq \tau-|\xi-\eta|-|\eta| \leq$ $\left.1,\left|\xi_{1}-\eta_{1}\right|^{1+\varepsilon}<\xi_{2},\left|\eta_{2}\right|<1, \eta_{1}>0\right\}$ so that $R$ has measure comparable to 1 . Since the integrand is everywhere nonnegative, we have

$$
\alpha{\widehat{u}+v_{+}}(\tau, \xi) \gtrsim \frac{1}{\langle\xi\rangle^{s+1+\varepsilon}} \frac{1}{\langle\xi\rangle^{s+1 / 2+\varepsilon}} \int_{R} d \eta \gtrsim \frac{1}{\langle\xi\rangle^{2 s+3 / 2+2 \varepsilon}} .
$$

\section{UPPER BOUNDS FOR DOUBLE INTERACTION}

Upper bound for $\alpha{\widetilde{u_{+}}}_{v_{+}}$. We are interested in estimates near the line segment in $\left(\xi_{1}, \xi_{2}\right)$ connecting $(\tau, 0)$ to $(0, \tau)$, but away from the endpoints (thus $|\xi| \approx\left|\xi_{1}\right| \approx\left|\xi_{2}\right|$ here). Note that the integral below is rapidly decreasing in cones away from this line segment. From above,

$$
\alpha{\widehat{\tilde{u}}+v_{+}}_{1}\left(\tau, \xi_{1}, \xi_{2}\right)=\int \hat{\alpha}(\tau-|\xi-\eta|-|\eta|) \hat{f}(\xi-\eta) \hat{g}(\eta) d \eta .
$$

We have $\hat{\alpha} \leq c$ for some constant $c$. Moreover, on the support of the integrand,

$$
\hat{f}(\xi-\eta) \leq \frac{1}{\left\langle\xi_{2}-\eta_{2}\right\rangle^{s+1+\varepsilon}} \lesssim \frac{1}{\langle\xi\rangle^{s+1+\varepsilon}}
$$

since $\hat{g}$ is supported where $\left|\eta_{2}\right| \leq 1$. Similarly, $|\eta| \approx\left|\eta_{1}\right|$ on $\operatorname{supp} \hat{g}$, and

$$
\left\langle\eta_{1}\right\rangle \leq\left\langle\xi_{1}-\eta_{1}\right\rangle+\left\langle\xi_{1}\right\rangle \lesssim\left\langle\xi_{2}-\eta_{2}\right\rangle+\left\langle\xi_{2}\right\rangle \leq\left\langle\xi_{2}\right\rangle \text { on supp } \hat{f} \text {. }
$$



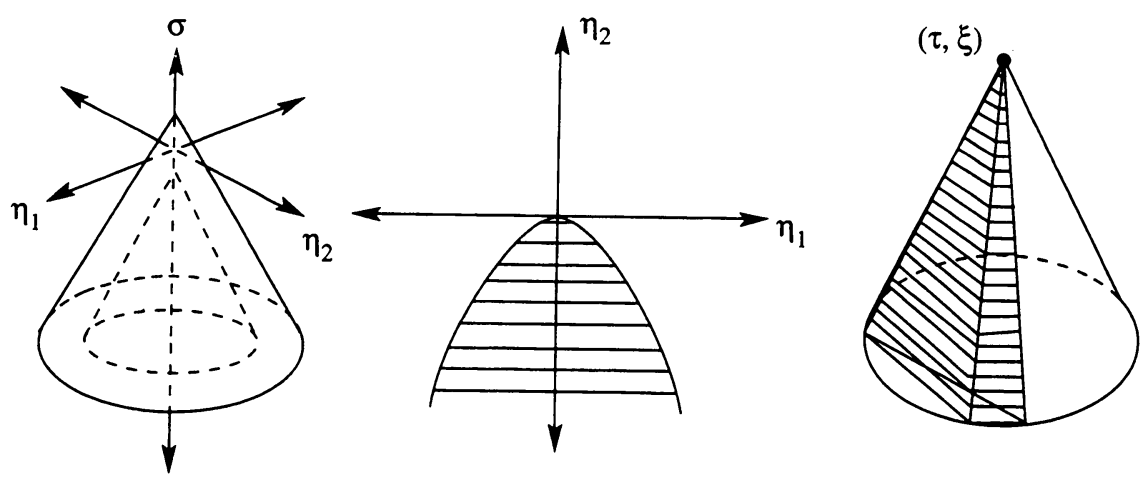

FIGURE 4. Support for $\hat{\alpha}(\sigma+|\eta|), \hat{f}(-\eta), \alpha{\widehat{\tilde{u}}+v_{+}}(\tau-\sigma, \xi-\eta)$

Thus,

$$
\hat{g}(\eta) \leq \frac{1}{\langle\eta\rangle^{s+1 / 2+\varepsilon}} \lesssim \frac{1}{\langle\xi\rangle^{s+1 / 2+\varepsilon}} .
$$

By the rapid decrease of $\hat{\alpha}$, we can assume that $|\tau-| \xi-\eta|-| \eta|| \leq|\xi|^{\delta}$. From $\S 4$, this ellipse in $\left(\eta_{1}, \eta_{2}\right)$ has thickness $|\xi|^{\delta}$, and for the values of $\left(\xi_{1}, \xi_{2}\right)$ under consideration, it intersects supp $\hat{g}$ in a set $S$ of size $|\xi|^{\delta}$. See Figure 2. Therefore for these values of $\left(\xi_{1}, \xi_{2}\right)$,

$$
\alpha{\widehat{\tilde{u}}+v_{+}} \lesssim \frac{1}{\langle\xi\rangle^{2 s+3 / 2+2 \varepsilon}} \int_{S} d \eta \lesssim \frac{\langle\xi\rangle^{\delta}}{\langle\xi\rangle^{2 s+3 / 2+2 \varepsilon}}=\frac{1}{\langle\xi\rangle^{2 s+3 / 2+2 \varepsilon-\delta}} .
$$

\section{LOWER BOUNDS FOR TRIPLE INTERACTION}

From $\S 4$,

$$
\alpha^{2}{\widehat{\tilde{u}}+\tilde{u}_{-}}_{v_{+}}\left(\tau, \xi_{1}, \xi_{2}\right)=\int \alpha{\widehat{\tilde{u}}+v_{+}}_{(\tau-\sigma, \xi-\eta) \hat{\alpha}(\sigma+|\eta|) \hat{f}(-\eta) d \eta}
$$

By the rapid decrease of $\hat{\alpha}$, we can concentrate our attention on those $(\sigma, \eta)$ with $|\sigma+| \eta||<1$. Assume $\hat{\alpha}(\sigma+|\eta|)$ has bounds from below where $-1 \leq$ $\sigma+|\eta| \leq 1$, a thickened cone. We know that $\hat{f}(-\eta)$ has support for $\eta_{2}<$ $0,-\left|\eta_{2}\right| \leq \eta_{1}^{1+\varepsilon} \leq\left|\eta_{2}\right|$.

We can assume that $\alpha \widehat{u}_{+} \widehat{v}_{+}(\tau-\sigma, \xi-\eta)$ has support in a wedge within a cone which opens downward, with vertex $(\tau, \xi)$. See Figure 4 . This is the same wedge that was found in $\S 4$, transposed.

We need to find bounds from below on $\alpha^{2} \widehat{\tilde{u}}_{+} \widehat{\tilde{u}}_{-} v_{+}$for $\left(\tau, \xi_{1}, \xi_{2}\right)=(\tau,-\tau, 0)$, $\tau>0$ (as in Figure 5). Again, it is assumed that $\left(\xi_{1}, \xi_{2}\right)$, as well as $\left(\xi_{1}-\eta_{1}, \xi_{2}-\right.$ $\left.\eta_{2}\right)$, lie in a conic set away from the directions $(1,0)$ and $(0,1)$.

$$
\alpha^{2} \widehat{\tilde{u}_{+} \tilde{u}_{-}} v_{+}\left(\tau, \xi_{1}, \xi_{2}\right)=\int \alpha \widehat{\tilde{u}}_{+} \widehat{v}_{+}(\tau-\sigma, \xi-\eta) \hat{\alpha}(\sigma+|\eta|) \hat{f}(-\eta) d \eta
$$

In the support of the integrand, $|(\sigma, \eta)|$ must be large compared to $|(\tau, \xi)|$ so that the circles $(\sigma+|\eta|=0,|\tau-\sigma|=|\xi-\eta|$, for fixed $\sigma<0)$ are close enough to allow a triple intersection of the regions involved. So $\langle\xi-\eta\rangle \approx\langle\eta\rangle \approx\langle(\sigma, \eta)\rangle$.

$$
\alpha{\widehat{\tilde{u}}+v_{+}}(\tau-\sigma, \xi-\eta) \gtrsim \frac{1}{\langle\xi-\eta\rangle^{2 s+3 / 2+\varepsilon}} \approx \frac{1}{\langle(\sigma, \eta)\rangle^{2 s+3 / 2+\varepsilon}} .
$$


Fixed $\sigma<0$ :

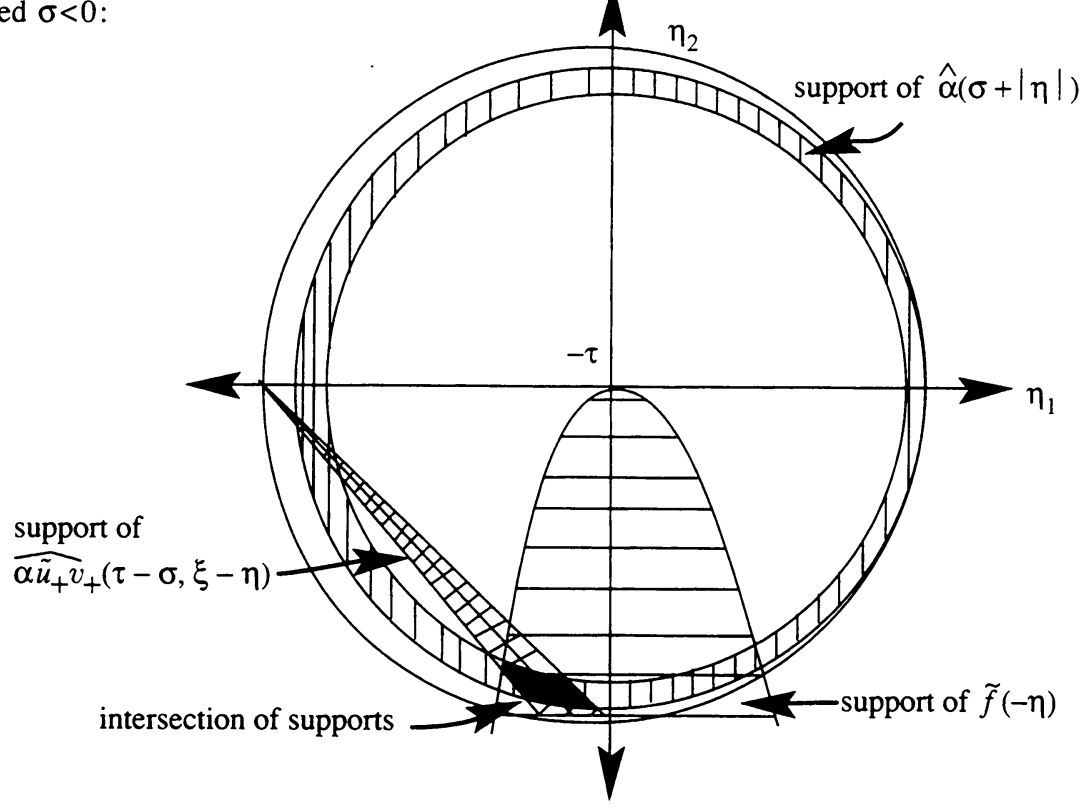

FIGURE 5. Region of support for triple intersection

For $(\xi-\eta)$ in the triangular wedge determined by $(\tau-\sigma)$ as described in $\S 4$, we can assume $\hat{\alpha}(\sigma+|\eta|) \geq 1$ for $|\sigma+| \eta|| \leq 1$. Moreover, for such $(\sigma, \eta)$, and on a conic neighborhood of $(0,-1)$ in $\left(\eta_{1}, \eta_{2}\right)$,

$$
\hat{f}(-\eta) \geq 1 /\left\langle\eta_{2}\right\rangle^{s+1+\varepsilon} \approx 1 /\langle\sigma, \eta\rangle^{s+1+\varepsilon}
$$

for $\eta$ in the region where the integrand has bounds from below.

Claim. We have a triple intersection of the sets described above for $|\sigma| \geq \tau^{1+\varepsilon}$, and for fixed large $\sigma$,

$$
-|\sigma|^{-1 /(1+\varepsilon)} \lesssim \eta_{1}+\tau \lesssim|\sigma|^{1 /(1+\varepsilon)}=|\sigma|^{1-\varepsilon /(1+\varepsilon)} .
$$

Proof. The $\eta_{1}$ bounds come from the width of the wedge described above (see Figure 5). Since we are always looking at the wide end of the wedge, the width of the wedge in the intersection $\geq(1 / 2)|\sigma|^{1 /(1+\varepsilon)}$. For $|\sigma|=\tau^{1+\varepsilon}$, we have the situation which is illustrated in Figure 5.

So, by the nonnegativity of the integrand,

$$
\begin{aligned}
\alpha^{2} \widehat{\tilde{u}_{+} \tilde{u}_{-}} v_{+} & \geq \int_{-\infty}^{-\tau^{1+\varepsilon}} \int_{-|\sigma|^{1-\varepsilon /(1+\varepsilon)}}^{|\sigma|^{1+\varepsilon /(1+\varepsilon)}} \int \frac{d \eta_{2} d \eta_{1} d \sigma}{\langle\sigma, \eta\rangle^{3 s+5 / 2+\varepsilon}} \\
& \gtrsim \frac{|\xi|^{\delta}}{\langle\tau\rangle^{3 s+1 / 2+\varepsilon^{\prime}}} \gtrsim \frac{1}{\langle\xi\rangle^{3 s+1 / 2+\varepsilon^{\prime}-\delta}},
\end{aligned}
$$

where $\varepsilon^{\prime} \rightarrow 0$ as $\varepsilon \rightarrow 0$. ( $\left(|\xi|^{\delta}\right.$ comes from $\eta_{2}$ integration.)

7. UPPER BOUNDS FOR TRIPLE INTERACTION

As before,

$$
\alpha^{2} \widehat{\tilde{u}_{+}} \tilde{u}_{-} v_{+}\left(\tau, \xi_{1}, \xi_{2}\right)=\int \alpha \widetilde{u}_{+} v_{+}(\tau-\sigma, \xi-\eta) \hat{\alpha}(\sigma+|\eta|) \hat{f}(-\eta) d \eta .
$$




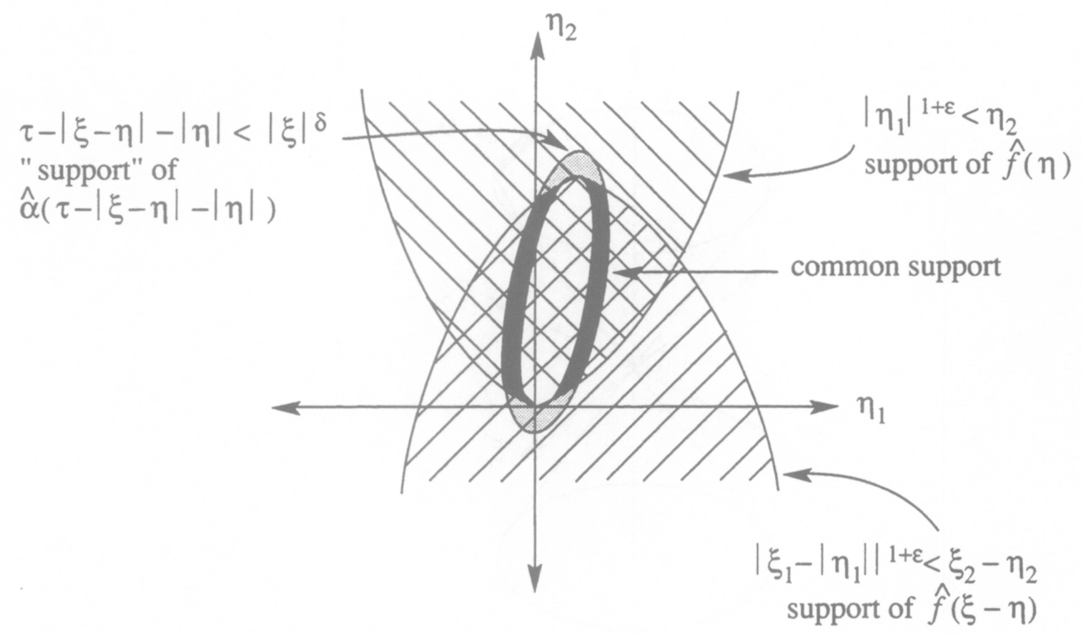

FIGURE 6. Bounded regions for double interaction

Here, once again, $|\sigma| \gtrsim \tau^{1+\varepsilon}$ in the region of triple intersection. Since $\eta \in$ supp $f(-\eta) \subset\left\{\eta_{2} \leq\left|\eta_{1}\right|\right\}$, we can use this fact, along with some elementary trigonometry to obtain a constant $c, 0<c<1$, such that $|\eta| \leq c|\sigma|+\tau \lesssim|\sigma|$, the last inequality holding for sufficiently large $\tau$.

$$
\alpha{\widehat{\tilde{u}}+v_{+}}_{+}(\tau-\sigma, \xi-\eta) \leq 1 /\langle\xi-\eta\rangle^{2 s+3 / 2+2 \varepsilon-\delta} \lesssim 1 /\langle\sigma, \eta\rangle^{2 s+3 / 2+2 \varepsilon-\delta},
$$

in the region of triple intersection. Moreover,

$$
\hat{\alpha}(\sigma+|\eta|) \lesssim 1 \text { and } \hat{f}(-\eta) \leq 1 /\left\langle\eta_{2}\right\rangle^{s+1+\varepsilon} \approx 1 /\langle\sigma, \eta\rangle^{s+1+\varepsilon},
$$

in the region of triple intersection. So

$$
\alpha^{2} \widehat{\tilde{u}_{+} \tilde{u}_{-}} v_{+} \leq \int_{-\infty}^{-\tau^{1+\varepsilon}} \int_{-|\sigma|}^{|\sigma|} \int \frac{d \eta_{2} d \eta_{1} d \sigma}{\langle\sigma, \eta\rangle^{3 s+5 / 2+3 \varepsilon-\delta}} \lesssim \frac{1}{\langle\xi\rangle^{3 s+1 / 2+\varepsilon^{\prime}}}
$$

where $\varepsilon^{\prime}$ is small and $\varepsilon^{\prime} \rightarrow 0$ as $\varepsilon \rightarrow 0$. So $\alpha^{2} \widehat{u}_{+} \widehat{\tilde{u}}_{-} v_{+} \in H_{m l}^{3 s-1-\varepsilon^{\prime \prime}}$ for $\left(\tau, \xi_{1}, \xi_{2}\right)=(\tau,-\tau, 0)$.

Note that these bounds from above will hold for other directions, as long as we stay away from the original singular directions of $v$, as well as the singular directions of $v^{2}$, resulting from the convolution of $\hat{v}$ with itself. (See Figure 6.)

\section{BOUNDS ON DOUBLE INTERACTION FOR THE PRIMARY TERM}

The other main term to be analyzed is the $\tilde{u}_{+}^{2} \tilde{u}_{-}$term. The $\tilde{u}_{+} \tilde{u}_{-}^{2}$ term will have similar bounds, and will not be considered separately. First, bounds on $\widehat{\alpha \tilde{u}_{+}^{2}}$ :

$$
\widehat{\alpha \tilde{u}_{+}^{2}}=\int \hat{\alpha}(\tau-|\xi-\eta|-|\eta|) \hat{f}(\xi-\eta) \hat{f}(\eta) d \eta .
$$

Bounds from below on $\widehat{\tilde{u}_{+}^{2}}$. As before, assume $\hat{\alpha} \geq 1$ for $|\tau-| \xi-\eta|-| \eta||<1$. We will find bounds from below in a slightly filled-in region of a small cone $C$ about the $\left(\tau, \xi_{1}, \xi_{2}\right)=(1,0,1)$ direction. The filled-in section of the cone 


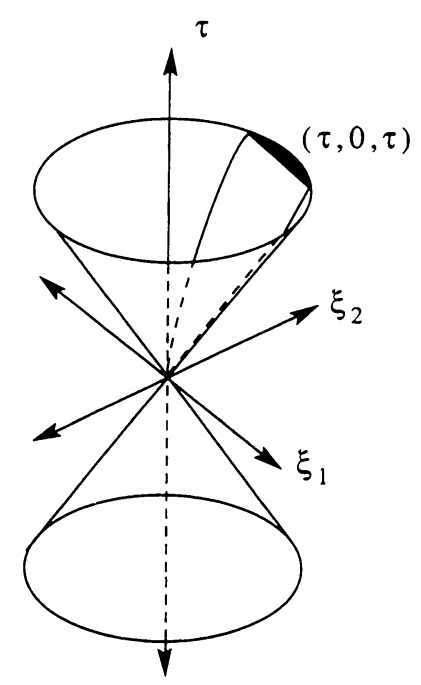

FIGURE 7. Region of lower bound for $\widehat{u_{+}^{2}}$

includes line segments connecting the points on the surface of the light cone where $\hat{f}(\xi)$ is supported. See Figure 7. This is in contrast to the fact that for $\tilde{u}_{+}$we could obtain bounds from below within only a fixed unit distance from the surface of the light cone. Notice that since the projection on the Fourier transform variables of the wave front set of $\tilde{u}_{+}^{2}$ is also a cone about the $(1,0,1)$ direction, outside of that cone $\widehat{\tilde{u}_{+}^{2}}$ is rapidly decreasing.

In $C$, where the integrand is bounded from below,

$$
\hat{\alpha}(\tau-|\xi-\eta|-|\eta|) \geq 1 \text {. }
$$

Because $\left\langle\xi_{2}-\eta_{2}\right\rangle \leq\left\langle\xi_{2}\right\rangle \approx\langle\xi\rangle$ in the support of the integrand,

$$
\hat{f}(\xi-\eta) \approx 1 /\left\langle\xi_{2}-\eta_{2}\right\rangle^{s+1+\varepsilon} \gtrsim 1 /\langle\xi\rangle^{s+1+\varepsilon} .
$$

Also, $\langle\eta\rangle \lesssim\langle\xi\rangle$, since $\langle\xi\rangle \approx \tau \approx|\xi+\eta|+|\eta|$, and so

$$
\hat{f}(\eta) \approx 1 /\langle\eta\rangle^{s+1+\varepsilon} \gtrsim 1 /\langle\xi\rangle^{s+1+\varepsilon} \text {. }
$$

So

$$
\widehat{\alpha \tilde{u}_{+}^{2}} \gtrsim \iint \frac{1}{\langle\xi\rangle^{2 s+2+2 \varepsilon}} d \eta \gtrsim \frac{1}{\langle\xi\rangle^{2 s+2+2 \varepsilon}}|\xi| \text { on } C \text {. }
$$

(The height of the support is $\gtrsim|\xi|$, while the width is $\gtrsim 1$ ). Thus

$$
\widehat{\alpha \tilde{u}_{+}^{2}} \gtrsim \frac{1}{\langle\xi\rangle^{2 s+1+2 \varepsilon}} \text { on } C \text {. }
$$

Bounds from above on $\widehat{\tilde{u}_{+}^{2}}$.

$$
\widehat{\alpha \tilde{u}_{+}^{2}}=\int \hat{\alpha}(\tau-|\xi-\eta|-|\eta|) \hat{f}(\xi-\eta) \hat{f}(\eta) d \eta .
$$

Now, we will find bounds from above in the cone $C$, where $\widehat{\tilde{u}_{+}^{2}}$ is not rapidly decreasing. Here, we consider bounds from above in terms of $\langle\tau-|\xi|\rangle$, because if we consider bounds only in terms of $\langle\xi\rangle$ or $\langle(\tau, \xi)\rangle$, we get only the original 
upper bound $c /\langle\xi\rangle^{s+1+\varepsilon}$, since $C$ contains $\Pi W F\left(\tilde{u}_{+}^{2}\right)$. Using $\langle\tau-|\xi|\rangle$, in effect, excises the "bad" direction. We have $\hat{\alpha}(\tau-|\xi-\eta|-|\eta|) \lesssim 1$.

Case 1: $\left\langle\xi_{2}-\eta_{2}\right\rangle \geq(1 / 2)\left\langle\xi_{\rangle}\right\rangle$.

$$
\hat{f}(\xi-\eta) \approx 1 /\left\langle\xi_{2}-\eta_{2}\right\rangle^{s+1+\varepsilon} \lesssim 1 /\langle\xi\rangle^{s+1+\varepsilon} .
$$

By the rapid decrease of $\hat{\alpha}$, we can assume that $|\tau-| \xi-\eta|-| \eta|| \leq|\xi|^{\delta}$ for $\delta>0$ small. Note that $\tau-|\xi-\eta|-|\eta| \geq-|\xi|^{\delta}$, and so $\tau \geq|\xi-\eta|+|\eta|-|\xi|^{\delta} \geq|\xi|-|\xi|^{\delta}$. Therefore $|\xi|-\tau \leq|\xi| \delta$.

If $|\tau-| \xi|| \leq 2|\xi|^{\delta}$, then $|\xi|^{\delta} /\langle\tau-|\xi|\rangle \geq 1$, and we estimate

$$
\frac{1}{\langle\eta\rangle^{s+1+\varepsilon}} \lesssim 1 \lesssim \frac{|\xi|^{\delta}}{\left\langle\tau-\left.|\xi|\right|^{s+1+\varepsilon}\right.}
$$

If $|\tau-| \xi|| \geq 2|\xi|^{\delta}$, then since $|\xi|-\tau \leq|\xi|^{\delta}$ from above, we must have $\tau-|\xi| \geq$ $2|\xi|^{\delta}$. Thus, since

$$
|\eta| \geq \tau-|\xi-\eta|-|\xi|^{\delta} \geq \tau-(|\eta|+|\xi|)-|\xi|^{\delta},
$$

we have

$$
2|\eta| \geq \tau-|\xi|-|\xi|^{\delta} \geq(1 / 2)(\tau-|\xi|)=(1 / 2)|\tau-| \xi|| .
$$

In this case,

$$
\frac{1}{\langle\eta\rangle^{s+1+\varepsilon}} \lesssim \frac{1}{\langle\tau-|\xi|\rangle^{s+1+\varepsilon}}
$$

In either case, we have

$$
\hat{f}(\eta) \approx 1 /\langle\eta\rangle^{s+1+\varepsilon} \lesssim\langle\xi\rangle^{\delta} /\langle\tau-|\xi|\rangle^{s+1+\varepsilon} .
$$

Case 2: $\left\langle\xi_{2}-\eta_{2}\right\rangle \leq(1 / 2)\langle\xi\rangle$.

Then $\langle\eta\rangle \gtrsim\langle\xi\rangle$. Interchange the roles of $\eta$ and $\xi-\eta$ in Case 1 . In either case, we obtain

$$
\widehat{\alpha \tilde{u}_{+}^{2}} \lesssim \iint \frac{\langle\xi\rangle^{\delta}}{\langle\xi\rangle^{s+1+\varepsilon}\langle\tau-|\xi|\rangle^{s+1+\varepsilon}} d \eta \lesssim \frac{1}{\langle\xi\rangle^{s+1+\varepsilon}\langle\tau-|\xi|\rangle^{s+1+\varepsilon}}|\xi| \cdot|\xi|^{2 \delta} .
$$

(The height of the support is $\leq|\xi|$, while the width is $\lesssim|\xi|^{\delta}$, as in $\S 4$.) Thus

$$
\widehat{\alpha \tilde{u}_{+}^{2}} \lesssim \frac{1}{\langle\xi\rangle^{s+\varepsilon-2 \delta}\left\langle\tau-\left.|\xi|\right|^{s+1+\varepsilon}\right.} \text {. }
$$

\section{BOUNDS ON TRIPLE INTERACTION FOR THE PRIMARY TERM}

We focus on the direction $\left(\tau, \xi_{1}, \xi_{2}\right)=(\tau,-\tau, 0)$, and have

$$
\alpha^{2} \widehat{\tilde{u}_{+}^{2}} \tilde{u}_{-}=\int \widehat{\alpha \tilde{u}_{+}^{2}}(\tau-\sigma, \xi-\eta) \hat{\alpha}(\sigma+|\eta|) \hat{f}(-\eta) d \eta .
$$

The essential support of $\widehat{\alpha \tilde{u}_{+}^{2}}(\tau-\sigma, \xi-\eta)$ is a slightly filled-in section of an inverted cone as shown in Figure 8.

The essential support of $\hat{\alpha}(\sigma+|\eta|)$ is an inverted, thickened cone, as in Figure 9. $\hat{f}(-\eta)$ is supported in an inverted parabola: $-\left|\eta_{1}\right|^{1+\varepsilon} \geq \eta_{2}$.

The three sets above intersect when the thickness of the slightly filled-in section of the cone exceeds $\tau$ :

$$
(\tau-\sigma)^{1-2 \varepsilon /(1+\varepsilon)}>\tau
$$


$(\tau, \xi)$

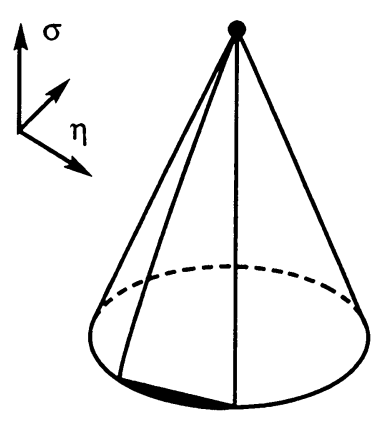

fixed $\sigma<0$ plane

radius $=\tau-\sigma$

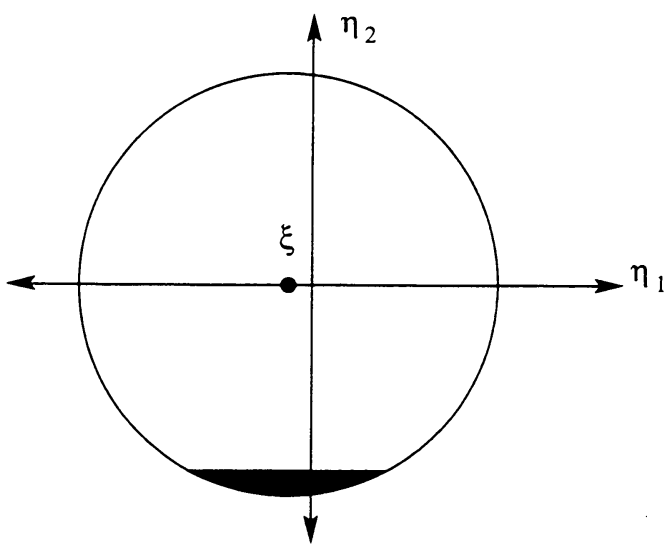

FIGURE 8. Support of $\widehat{\alpha \tilde{u}_{+}^{2}}(\tau-\sigma, \xi-\eta)$
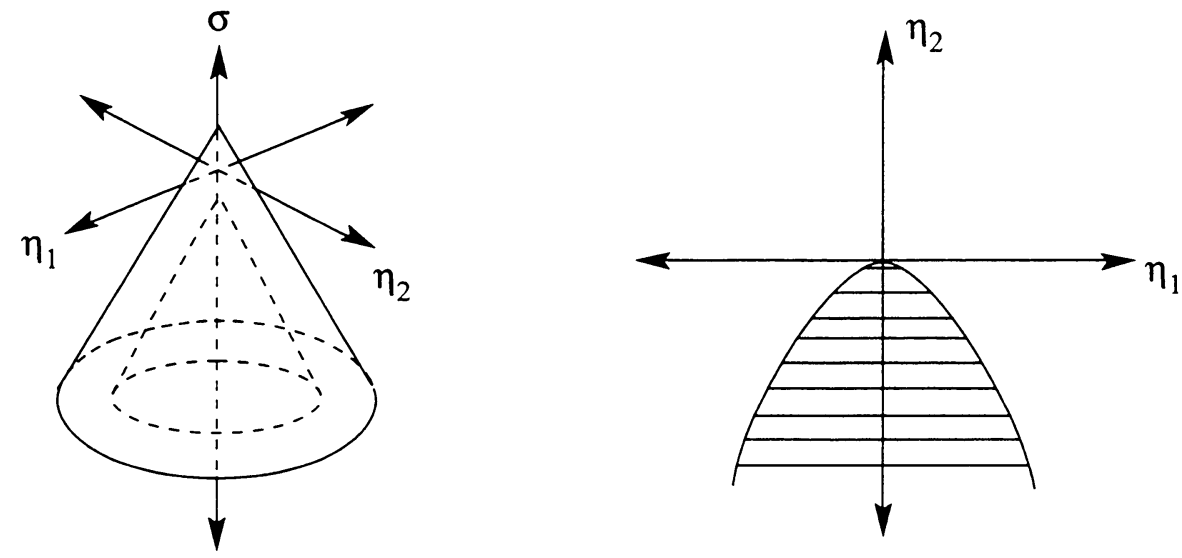

FIGURE 9. Support of $\hat{\alpha}(\sigma+|\eta|)$ and $\hat{f}(-\eta)$

i.e., $-\sigma \gtrsim \tau^{(1+\varepsilon) /(1-\varepsilon)}-\tau \gtrsim \tau^{(1+\varepsilon) /(1-\varepsilon)}$. Thus the $\sigma$ integration is over the interval from $-\tau^{(1+\varepsilon) /(1-\varepsilon)}$ to $-\infty$.

Bounds from below.

$$
\alpha^{2} \widehat{\tilde{u}_{+}^{2}} \tilde{u}_{-}=\int \widehat{\alpha \tilde{u}_{+}^{2}}(\tau-\sigma, \xi-\eta) \hat{\alpha}(\sigma+|\eta|) \hat{f}(-\eta) d \eta .
$$

$\hat{\alpha}(\sigma+|\eta|) \geq 1$ for $\sigma+|\eta| \leq 1$. The $\eta_{1}$ integration is over roughly the width of the parabola, so $\eta_{1} \approx \sigma^{1 /(1+\varepsilon)}$. (We can assume that the arc is straightened so that the $\eta_{2}$ integration is between two constants.) Consequently, the nonnegativity of the integral yields

$$
\alpha^{2} \widehat{\tilde{u}_{+}^{2}} \tilde{u}_{-}(\tau, \xi) \gtrsim \int_{-\infty}^{-\tau^{(1+\varepsilon) /(1-\varepsilon)}} \int_{\sigma_{1 /(1+\varepsilon)}}^{-\sigma^{1 /(1+\varepsilon)}} \int_{\sigma-1}^{\sigma+1} \frac{d \eta_{2} d \eta_{1} d \sigma}{\langle\sigma, \eta\rangle^{3 s+2+\varepsilon^{\prime}}} \approx \frac{1}{\langle\tau\rangle^{3 s+\varepsilon^{\prime \prime}}} .
$$

(see Figure 10.)

Bounds from above. By the rapid decrease of $\hat{\alpha}$, for upper bounds we can assume that $|\sigma+| \eta||<|\xi|^{\delta}$. Also, $\sigma<0, \tau>0$, and $|\xi-\eta|<\sigma+c \tau$ for some $c<1$, using trigonometry and the fact that the support of the integrand 
fixed $\sigma<0$

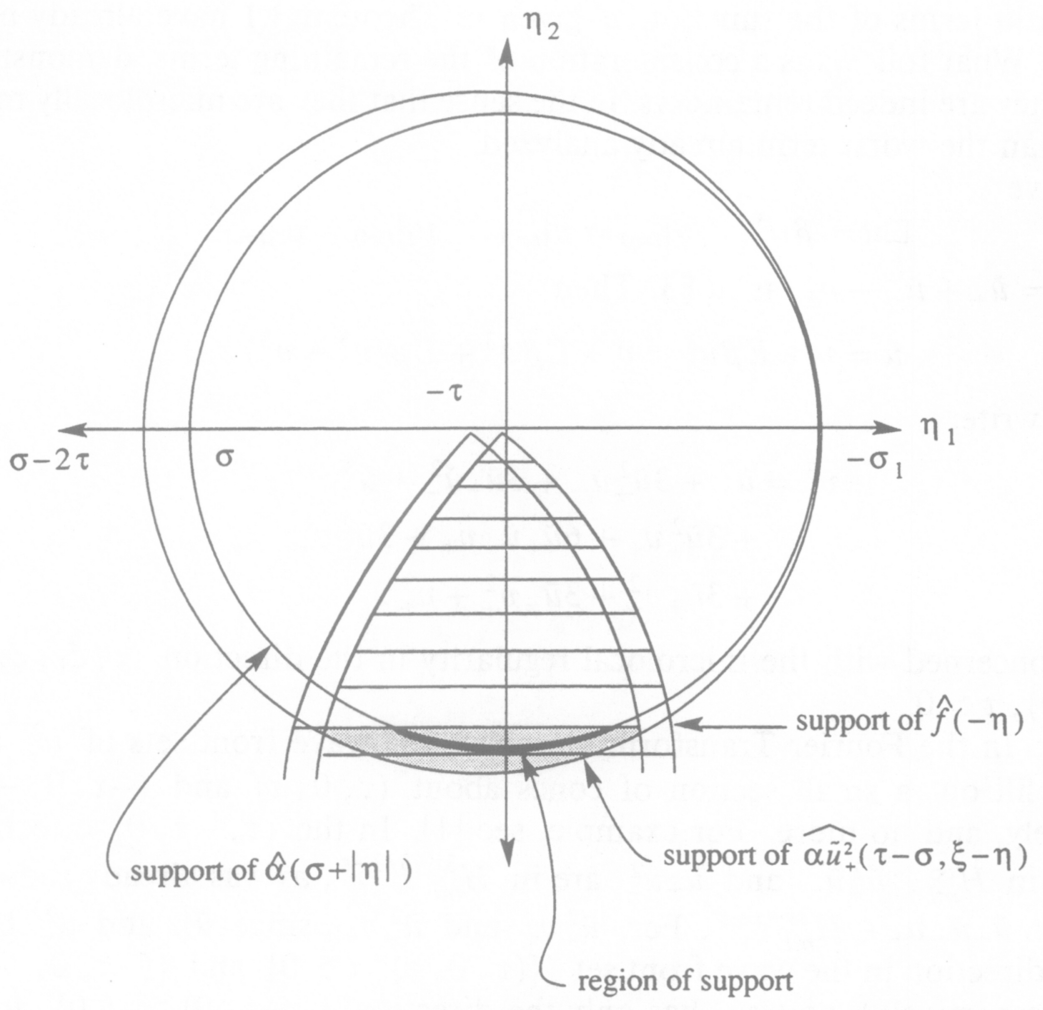

FIGURE 10. Support for triple intersection

lies below the lines $\eta_{1} \pm \eta_{2}=0$, and between the lines $\eta_{1}-\xi \pm \eta_{2}=0$, which allows us to conclude that $\tau-\sigma-|\xi-\eta| \gtrsim \tau$ for sufficiently large $\tau$. Therefore on this set

$$
\begin{aligned}
\widehat{\alpha^{2} \tilde{u}_{+}^{2}}(\tau-\sigma, \xi-\eta) & \lesssim \frac{1}{\langle\tau-\sigma-|\xi-\eta|\rangle^{s+1+\varepsilon}\langle\xi-\eta\rangle^{s+\varepsilon-2 \delta}} \\
\alpha^{2} \widehat{\tilde{u}_{+}^{2}} \tilde{u}_{-}(\tau, \xi) & \lesssim \int_{-\infty}^{-\tau^{(1+\varepsilon) /(1-\varepsilon)}} \int_{\sigma^{1 /(1+\varepsilon)}}^{-\sigma^{1 /(1+\varepsilon)}} \int_{\sigma-|\xi|^{\delta}}^{\sigma+|\xi|^{\delta}} \frac{d \eta_{2} d \eta_{1} d \sigma}{\langle\sigma, \eta\rangle^{2 s+1+2 \varepsilon-\delta}\langle\tau\rangle^{s+1+\varepsilon}} \\
& \approx \frac{|\xi|^{\delta}}{\langle\tau\rangle^{3 s+\varepsilon^{\prime \prime}-2 \delta}} .
\end{aligned}
$$

Thus

$$
\alpha^{2} \widetilde{u}_{+}^{2} \tilde{u}_{-}(\tau, \xi) \in H_{m l}^{3 s-3 / 2-\varepsilon^{\prime \prime}}
$$

in the direction $(\tau,-\tau, 0)$, but

$$
\alpha^{2} \widehat{u}_{+}^{2} \tilde{u}_{-}(\tau, \xi) \notin H_{m l}^{3 s-3 / 2-\varepsilon^{\prime \prime}+\delta}
$$

for any $\delta>0$. Note that this term is less regular than the $\alpha^{2} \widehat{u}_{+} \widehat{u}_{-} v_{+}$term. So

$$
v^{3}=\left(\tilde{u}_{+}+\tilde{u}_{-}+v_{+}\right)^{3} \in H_{m l}^{3 s-3 / 2-\varepsilon^{\prime \prime}}, \quad v^{3} \notin H_{m l}^{3 s-3 / 2-\varepsilon^{\prime \prime}+\delta},
$$




\section{ANALYSIS OF THE REMAINDER TERMS}

The main terms of the function $u$ given in Theorem 3.1 have already been analyzed. What follows is a consideration of the remaining terms, demonstrating that they are indeed remainders, in the sense that they are microlocally more regular than the worst term already analyzed.

We solve

$$
\square u=\beta u^{3},\left.\quad u\right|_{t=0}=\left.v\right|_{t=0},\left.\quad u_{t}\right|_{t=0}=\left.v_{t}\right|_{t=0}
$$

where $v=\tilde{u}_{+}+\tilde{u}_{-}+v_{+}$as in $\S 3$. Then

$$
u=v+E \beta u^{3}=v+E \beta v^{3}+E \beta\left(u^{3}-v^{3}\right) .
$$

First, we write:

Line 1

$$
\begin{aligned}
v^{3}= & \tilde{u}_{+}^{3}+3 \tilde{u}_{+}^{2} \tilde{u}_{-}+3 \tilde{u}_{+} \tilde{u}_{-}^{2}+\tilde{u}^{3} \\
& +3 \tilde{u}_{+}^{2} v_{+}+6 \tilde{u}_{+} \tilde{u}_{-} v_{+}+3 \tilde{u}_{-}^{2} v_{+} \\
& +3 \tilde{u}_{+} v_{+}^{2}+3 \tilde{u}_{-} v_{+}^{2}+v_{+}^{3} .
\end{aligned}
$$

Line 2

Line 3

We are concerned with the microlocal regularity in the direction $\left(\tau, \xi_{1}, \xi_{2}\right)=$ $(\tau,-\tau, 0), \tau>0$.

Line 1: In the Fourier Transform variables, the wave front sets of $\tilde{u}_{+}^{3}$ and $\tilde{u}_{-}^{3}$ each fill out a small section of cones about $(\tau, 0, \tau)$ and $(-\tau, 0,-\tau)$, respectively, and no more. For example, see [1]. In the $(\tau,-\tau, 0)$ direction, these are in $H_{m l}^{\infty} . \tilde{u}_{+}^{2} \tilde{u}_{-}$and $\tilde{u}_{+} \tilde{u}_{-}^{2}$ are in $H_{m l}^{3 s-3 / 2-\varepsilon}$, as was already shown.

Line 2: $\tilde{u}_{+} \tilde{u}_{-} v_{+} \in H_{m l}^{3 s-1-\varepsilon}$. For, $\tilde{u}_{+}^{2} v_{+}$and $\tilde{u}_{-}^{2} v_{+}$, since $\tilde{u}_{+}^{2}$ and $\tilde{u}_{-}^{2}$ have only one direction in the wave front set $(\{(\tau, 0, \tau), \tau>0\}$ and $\{(-\tau, 0,-\tau)$, $\tau>0\}$, respectively), and $v_{+}$has only the direction $\{(\tau, \tau, 0), \tau>0\}, \tilde{u}_{+}^{2} v_{+}$ and $\tilde{u}_{-}^{2} v_{+}$have only the wedges $\left\{\left(\xi_{1}+\xi_{2}, \xi_{1}, \xi_{2}\right): \xi_{1} \geq 0, \xi_{2} \geq 0,\left(\xi_{1}, \xi_{2}\right) \neq\right.$ $(0,0)\}$ and $\left\{\left(\xi_{1}+\xi_{2}, \xi_{1}, \xi_{2}\right): \xi_{1} \geq 0, \xi_{2} \leq 0,\left(\xi_{1}, \xi_{2}\right) \neq(0,0)\right\}$. So these terms are in $H_{m l}^{\infty}$ in the $\{(\tau,-\tau, 0), \tau>0\}$ direction.

Line 3: Since $v_{+}^{2}$ is still conormal, it is microlocally in $H^{\infty}$ in the $(\tau,-\tau, 0)$ direction. $v_{+}^{3}$ is still conormal, and $v_{+}^{2} \tilde{u}_{ \pm}$has only one of the wedges described above in the $\Pi_{\xi} W F$ set.

We conclude that, in the $(\tau,-\tau, 0)$ direction, $v^{3} \in H_{m l}^{3 s-3 / 2-\varepsilon}$.

Next we write

$$
E \beta\left(u^{3}-v^{3}\right)=3 E \beta\left(v^{2} E \beta u^{3}\right)+3 E \beta\left(v\left(E \beta u^{3}\right)^{2}\right)+E \beta\left(E \beta u^{3}\right)^{3} .
$$

We will use the spaces in Definition 2.6 with $\omega_{1}=(\tau, 0, \tau), \omega_{2}=(-\tau, 0$, $-\tau)$, and $\omega_{3}=(\tau, \tau, 0), \tau>0$.

By Theorem 2.7 and Lemma 2.8, since $u \in H^{s, s, s}, v \in H^{s, \infty, \infty}$, and $u=$ $v+E \beta u^{3}$, it follows that $u \in H^{s, s+2, s+1}$, and inductively that

$$
\begin{gathered}
u \in H^{s, 2 s-1-\varepsilon, 3 s-2-\varepsilon}, \quad u^{2} \in H^{s, 2 s-1-\varepsilon, 3 s-2-\varepsilon}, \quad u^{3} \in H^{s, 2 s-1-\varepsilon, 3 s-2-\varepsilon} ; \\
E \beta u^{2},\left(E \beta u^{3}\right)^{2},\left(E \beta u^{3}\right)^{3} \in H^{s+1,2 s+1-\varepsilon, 3 s-1-\varepsilon} .
\end{gathered}
$$

Also, $v \in H^{s, \infty, \infty}, v^{2} \in H^{s, 2 s-1-\varepsilon, \infty}, v^{3} \in H^{s, 2 s-1-\varepsilon, 3 s-2-\varepsilon}$.

By the analysis above, since the set $G$ corresponds to the location where microlocal bounds on the singularities were obtained, we have the better estimate

$$
v^{3} \in H^{s, 2 s-1-\varepsilon, 3 s-3 / 2-\varepsilon} \text {. }
$$


Also, Theorem 2.7 yields

$$
v\left(E \beta u^{3}\right)^{2} \in H^{s, 2 s-\varepsilon, 3 s-1-\varepsilon}, \quad v^{2}\left(E \beta u^{3}\right) \in H^{s, 2 s-1-\varepsilon, 3 s-1-\varepsilon} .
$$

Finally we conclude that $E \beta\left(v\left(E \beta u^{3}\right)^{2}\right) \in H^{s+1,2 s+2-\varepsilon, 3 s-\varepsilon}, E \beta\left(v^{2}\left(E \beta u^{3}\right)\right) \in$ $H^{s+1,2 s+1-\varepsilon, 3 s-\varepsilon}, E \beta\left(\left(E \beta u^{3}\right)^{3}\right) \in H^{s+2,2 s+3-\varepsilon, 3 s-\varepsilon}$.

Now, $E \beta v^{3} \in H^{s+1,2 s+1-\varepsilon, 3 s-1 / 2-\varepsilon}$, and the bounds from below on the Fourier transform of $\beta v^{3}$ yield $\beta v^{3} \notin H_{m l}^{3 s-3 / 2-\varepsilon+\delta}$ for any $\delta>0$. It follows easily as in [1] that $E \beta v^{3} \notin H_{m l}^{3 s-1 / 2-\varepsilon+\delta}$.

Since

$$
u=v+E \beta v^{3}+3 E \beta\left(v^{2}\left(E \beta u^{3}\right)\right)+3 E \beta\left(v\left(E \beta u^{3}\right)^{2}\right)+E \beta\left(E \beta u^{3}\right)^{3},
$$

overall,

$$
u-\left(v+E \beta v^{3}\right) \in H^{s+1,2 s+1,3 s-\varepsilon} .
$$

Thus, $u-\left(v+E \beta v^{3}\right) \in H_{m l}^{3 s-\varepsilon}(\Gamma)$, where $\Gamma$ represents the point $\left(t, x, y ; \tau, \xi_{1}\right.$, $\left.\xi_{2}\right)=(0,0,0 ; 1,-1,0)$. Since $v \in H_{m l}^{\infty}(\Gamma)$, and we have seen that $E \beta v^{3} \notin$ $H_{m l}^{3 s-1 / 2-\varepsilon+\delta}(\Gamma)$, it follows that $u \notin H_{m l}^{3 s-1 / 2-\varepsilon+\delta}(\Gamma)$.

Our proof also shows that the interaction of the singularities conormal with respect to the pair $\{y=0\},\{x=y=0\}$ is in itself responsible for the appearance of nonlinear singularities.

Corollary 10.1. Let $s, \beta, \tilde{u}_{+}$and $\tilde{u}_{-}$be as in Theorem 3.1. Let $v=\tilde{u}_{+}+\tilde{u}_{-}$, and suppose that $u$ solves $\square u=\beta u^{3},\left.u\right|_{t=0}=\left.v\right|_{t=0},\left.u_{t}\right|_{t=0}=\left.v_{t}\right|_{t=0}$. Then $u$ has new singularities on the surface of the light cone, and in particular, $u \notin$ $H_{m l}^{3 s-1 / 2-\varepsilon+\delta}(r, r, 0 ; \tau,-\tau, 0), r \geq 0$.

Proof. The microlocal analysis of $\beta v^{3}$ above, on a neighborhood of $\{(t, x, y)$ $=(0,0,0)\}$, yields new singularities for $E \beta v^{3}$ on the corresponding nullbicharacteristic by Hörmander's Theorem. The remaining terms in the expression for $u$ are strictly smoother microlocally over $\{(t, x, y)=(0,0,0)\}$, and hence, again by Hörmander's Theorem, are strictly smoother on the nullbicharacteristics.

\section{MUlTIPLICATION OF CONORMAL DISTRIBUTIONS}

In the previous sections, we demonstrated that for certain conormal initial data, a spreading of singularities can occur compared to the corresponding linear problem. In what follows, we analyze conditions for which no new singularities appear. This section contains results on the multiplication of conormal distributions, which will be needed for this analysis. The proofs involve standard arguments. For details, see Holt [9]. Similar arguments appear in Rauch-Reed [14].

The following result treats microlocal regularity of the product of two conormal distributions, away from the wavefront sets of the two distributions. Regularity of type $H^{s_{1}}$ and $H^{s_{2}}$ in the original microlocal directions yields regularity of type $H^{s_{1}+s_{2}}$ in the directions due to interaction. This contrasts with Rauch's Lemma, where the distributions are not necessarily conormal, and the microlocal regularity is $H^{s_{1}+s_{2}-n / 2-\varepsilon}$ in the new directions. Similar results in a different context appear in Rauch-Reed [14]. 
Theorem 11.1. Given $u_{i} \in H^{s_{i}}\left(\mathbf{R}^{n}\right)$ conormal with respect to $x_{i}=0, i=1,2$, then $u_{1} u_{2} \in H_{m l}^{s_{1}+s_{2}}$, away from the wavefront sets of $u_{1}$ and $u_{2}$.

Proof. Although the theorem is true for $u_{i}$ a function of $n$ variables, it will be demonstrated in $\mathbf{R}^{2}$, since the $\mathbf{R}^{n}$ case can be easily reduced to the 2 variable case.

Since $u_{1} \in H^{s_{1}}\left(\mathbf{R}^{2}\right),\langle\xi\rangle^{s_{1}} \hat{u} \in L^{2}$. Since $u_{1}$ is conormal with respect to $x_{1}=0,\left\langle\xi_{2}\right\rangle^{k} \hat{u}_{1} \in L^{2}$ for any $k \geq 0$. So without loss of generality, $u_{1}$ can be regarded as being of the form

$$
\hat{u}_{1}(\xi)=\frac{f(\xi)}{\left\langle\xi_{2}\right\rangle^{k}\left\langle\xi_{1}\right\rangle^{s_{1}}},
$$

with $f \in L^{2}$, and for $k$ as large as we want. Similarly,

$$
\hat{u}_{2}(\xi)=\frac{g(\xi)}{\left\langle\xi_{1}\right\rangle^{k}\left\langle\xi_{2}\right\rangle^{s_{2}}},
$$

with $g \in L^{2}$. We wish to show that $\chi(\xi) u_{1} u_{2} \in H^{s_{1}+s_{2}}\left(\mathbf{R}^{2}\right)$, for $\chi(\xi)$ a cut-off function with conic support where $\xi_{1} \neq 0$ and $\xi_{2} \neq 0$, i.e.,

$$
\chi(\xi)\langle\xi\rangle^{s_{1}+s_{2}} \widehat{u_{1} u_{2}} \in L^{2} \text {. }
$$

We have

$$
\chi(\xi)\langle\xi\rangle^{s_{1}+s_{2}} \widehat{u_{1} u_{2}}(\xi)=\chi(\xi)\langle\xi\rangle^{s_{1}+s_{2}} \int \frac{f(\xi-\eta) g(\eta) d \eta_{1} d \eta_{2}}{\left\langle\xi_{2}-\eta_{2}\right\rangle^{k}\left\langle\xi_{1}-\eta_{1}\right\rangle^{s_{1}}\left\langle\eta_{1}\right\rangle^{k}\left\langle\eta_{2}\right\rangle^{s_{2}}} .
$$

To show that the above function is in $L^{2}$, we will use the following property (see e.g. [4]).

Lemma 11.2. Let $w(\xi)=\int F(\xi, \eta) f(\xi-\eta) g(\eta) d \eta$, where $f, g \in L^{2}$. Then $\|w\|_{2} \leq c\|f\|_{2}\|g\|_{2}$, as long as $F$ can be written as a sum of finitely many functions $F_{i}$ with

$$
\sup _{\xi} \int\left|F_{i}\right|^{2} d \eta<\infty \text { or } \sup _{\eta} \int\left|F_{i}\right|^{2} d \eta<\infty .
$$

So to show that $\chi(\xi)\langle\xi\rangle^{s_{1}+s_{2}} \widehat{u_{1} u_{2}}(\xi) \in L^{2}$, we apply the above lemma to $w(\xi)=\chi(\xi)\langle\xi\rangle^{s_{1}+s_{2}} \widehat{\chi u_{1} u_{2}}(\xi)=\int F(\xi, \eta) f(\xi-\eta) g(\eta) d \eta$, where

$$
F(\xi, \eta)=\frac{\chi(\xi)\langle\xi\rangle^{s_{1}+s_{2}}}{\left\langle\xi_{2}-\eta_{2}\right\rangle^{k}\left\langle\xi_{1}-\eta_{1}\right\rangle^{s_{1}}\left\langle\eta_{1}\right\rangle^{k}\left\langle\eta_{2}\right\rangle^{s_{2}}} .
$$

A straightforward argument demonstrates that

$$
\sup _{\xi} \int\left|F_{i}\right|^{2} d \eta<\infty
$$

as desired.

The following result treats the microlocal regularity of the product of a distribution with a conormal type singularity and a distribution with a singularity not of conormal type. The two distributions are singular in different microlocal directions. In the "wedge" generated by the singular directions on the Fourier Transform side, the regularity is $H^{s_{1}+s_{2}-1 / 2-\varepsilon}$. Note that there is a loss of $1 / 2$, rather than $n / 2$ as in Rauch's Lemma. In the other microlocal directions, the product is $C^{\infty}$. The proof uses an argument similar to the one used in the above theorem. 
Proposition 11.3. Let $v \in H^{s_{1}}\left(\mathbf{R}^{n}\right)$ be conormal with respect to a hyperplane, $s_{1}>1 / 2, s_{2} \geq 0$. Let $w \in H^{s_{2}}\left(\mathbf{R}^{n}\right)$ be such that $\Pi_{\xi} W F(v) \cap \Pi_{\xi} W F(w)=\varnothing$. Then

(a) $\chi_{K_{1}}(\xi)\langle\xi\rangle^{s_{1}+s_{2}-1 / 2-\varepsilon} \widehat{v w} \in L^{2}$, where $K_{1}$ is a conic set in closure $\left(\Pi_{\xi} W F(v)\right.$ $\left.+\Pi_{\xi} W F(w)\right)$, such that $K_{1} \cap \Pi_{\xi} W F(v)=K_{1} \cap \Pi_{\xi} W F(w)=\varnothing$.

(b) $\chi_{K_{2}}(\xi)\langle\xi\rangle^{m} \widehat{v w} \in L^{2}, \forall m>0$, where $K_{2}$ is a closed conic set contained in the complement of closure $\left(\Pi_{\xi} W F(v)+\Pi_{\xi} W F(w)\right)$.

Remark 11.4. For $v$ and $w$ as above, if $\Pi_{\xi} W F(w)$ is the ray $\left\{\xi_{2}>0, \xi_{1}=\right.$ $\left.\cdots=\xi_{n}=0\right\}$, then since in general $\Pi_{\xi} W F(v)$ is the line $\left\{\xi_{1} \neq 0, \xi_{2}=\cdots=\right.$ $\left.\xi_{n}=0\right\}$, the "wedge" is closure $\left(\Pi_{\xi} W F(w)+\Pi_{\xi} W F(v)\right)$. Rapid decrease holds for $\widehat{v w}$ outside the upper half-plane $\left\{\left(\xi_{1}, \xi_{2}, 0, \ldots, 0\right): \xi_{2} \geq 0,\left(\xi_{1}, \xi_{2}\right) \neq\right.$ $(0,0)\}$. If $v$ is further assumed to satisfy $\Pi_{\xi} W F(v)=\left\{\xi_{1}>0, \xi_{2}=\cdots=\right.$ $\left.\xi_{n}=0\right\}$, then closure $\left(\Pi_{\xi} W F(w)+\Pi_{\xi} W F(v)\right)=\left\{\left(\xi_{1}, \xi_{2}, 0, \ldots, 0\right): \xi_{1} \geq\right.$ $\left.0, \xi_{2} \geq 0,\left(\xi_{1}, \xi_{2}\right) \neq 0\right\}$, so in this case $\widehat{v w}$ has rapid decrease outside this quarter-plane.

The next result concerns a product, as before, of a conormal and nonconormal distribution, but with wavefront set in the same direction. Here the microlocal regularity away from the original direction is again $H^{s_{1}+s_{2}-1 / 2-\varepsilon}$. The proof is once again similar to those above.

Proposition 11.5. Let $v \in H^{s_{1}}\left(\mathbf{R}^{n}\right), s_{1}>1 / 2$, be conormal with respect to the plane $x_{1}=0$. Let $w \in H^{s_{2}}\left(\mathbf{R}^{n}\right), s_{2} \geq 0$, be such that $\Pi_{\xi} W F(w) \subset\{\xi$ : $\left|\left(\xi_{2}, \ldots, \xi_{n}\right)\right| \leq \varepsilon\left|\xi_{1}\right|$ for small $\left.\varepsilon, \xi_{1}>0\right\}$. Then away from the $\xi_{1}$ axis (i.e., $K$ is a cone in $\left.\mathbf{R}^{n} \backslash 0, K \cap \Pi_{\xi} W F(w)=\varnothing, K \cap \Pi_{\xi} W F(v)=0\right)$,

$$
\chi_{K}(\xi)\langle\xi\rangle^{s_{1}+s_{2}-1 / 2-\varepsilon} \widehat{v u} \in L^{2} .
$$

Remark 11.6. Note that Proposition 11.5 includes the case of $\Pi_{\xi} W F(w)$ in the positive $\xi_{1}$ direction and $\Pi_{\xi} W F(v)$ in the negative direction as a special case. If $\Pi_{\xi} W F(v)$ and $\Pi_{\xi} W F(w)$ are both in the same direction, there is nothing to check.

\section{NONSPREADING RESULT}

If, in $\S 3$, we had considered the linear solution $v=\tilde{u}_{+}+v_{+}+v_{-}$, where the first function is conormal with respect to $\{y=0, x=y=0\}$ and the other two conormal with respect to $\{x=0\}$, instead of $v$ consisting of the sum of two functions conormal with respect to $\{y=0, x=y=0\}$ and one conormal with respect to $\{x=0\}$, then no spreading of singularities compared to the linear case would occur for $u$ satisfying $\square u=f(u)$, where $f$ is a polynomial. We begin by defining microlocal spaces which are tailored to the geometry of this particular situation.

Definition 12.1. Define $H^{a, b, c, d}$ as follows, after multiplication by a $C_{0}^{\infty}\left(\mathbf{R} \times \mathbf{R}^{n}\right)$ cutoff function supported near $(0,0)$.

(1) If $K$ is a small conic neighborhood of the ray $R$ through $\left(1, \omega_{1}\right), \omega_{1} \in$ $S^{n-1}$, then $\chi_{K}(\tau, \xi)\langle(\tau, \xi)\rangle^{a} \hat{u} \in L^{2}$.

(2) If $\Gamma$ is a small tubular neighborhood of the line $L$ through $\left(1, \omega_{2}\right)$ and $\left(-1,-\omega_{2}\right), \omega_{2} \in S^{n-1}, \omega_{2} \neq \omega_{1}$, then $\chi_{\Gamma}(\tau, \xi)\langle(\tau, \xi)\rangle^{b} \hat{u} \in L^{2}$.

(3) If $B$ is a small conic neighborhood of a point $\left(\tau_{0}, \xi_{0}\right)$ in the open wedge $B^{\omega_{1}, \pm \omega_{2}}$ between $\left(1, \omega_{1}\right)$ and $\pm\left(1, \omega_{2}\right)$, then $\chi_{B}(\tau, \xi)\langle(\tau, \xi)\rangle^{c} \hat{u} \in L^{2}$. 


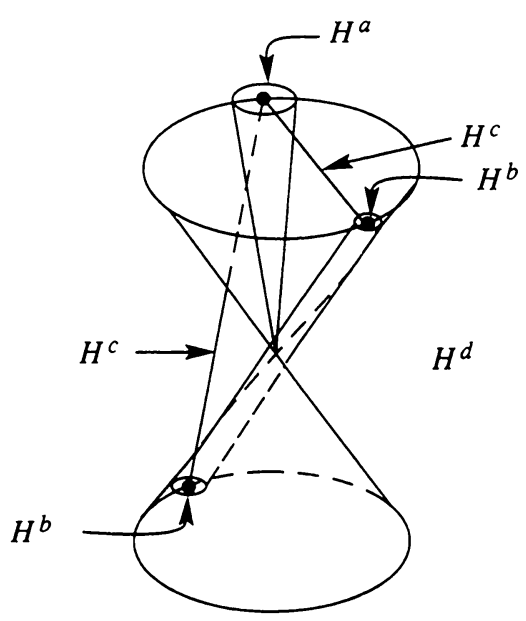

FIGURE 11. Spaces $H^{a, b, c, d}$

(4) If $G$ is a small conic neighborhood of $L$, then $\chi_{K \backslash \Gamma}(\tau, \xi)\langle(\tau, \xi)\rangle^{c} \hat{u} \in L^{2}$. Moreover, if $K$ is a small conic neighborhood of $\left(\tau_{1}, \xi_{1}\right) \in \mathbf{R}^{3} \backslash B^{+\omega_{1}, \pm \omega_{2}} \cup R \cup$ $L \cup\{0\}$, then $\chi_{G}(\tau, \xi)\langle(\tau, \xi)\rangle^{d} \hat{u} \in L^{2}$.

We will consider these spaces for $a$ and $b$ small, $c$ intermediate, and $d$ large. Thus the elements of $H^{a, b, c, d}$ will have restricted regularity near $R$ and $L$, and greatest regularity on conic sets avoiding the wedge generated by $R$ and $L$. Moreover, the intermediate regularity holds on a conic neighborhood of $L$, as long as the tubular neighborhood of $L$ is excised.

Lemma 12.2. If $u_{1}, u_{2} \in H^{a, b, c, d}$, then $u_{1} u_{2} \in H^{a, b, c, d}$, i.e., $H^{a, b, c, d}$ is an algebra, for $a>(n+1) / 2, b>1 / 2, a, b \leq c \leq d, c<a+b-1 / 2$. (See Figure 11.)

Proof. Consider $u_{i}, i=1,2$, of the form

$$
u_{i}=u_{i a}+u_{i b}+u_{i c}+u_{i d}
$$

where $\hat{u}_{i a}$ is supported in a cone about $\left(1, \omega_{1}\right),(K)$,

$\hat{u}_{i b}$ is supported in a tube about $\left(1, \omega_{2}\right),\left(-1,-\omega_{2}\right),(\Gamma)$,

$\hat{u}_{i c}$ is supported in a cone about a point in the wedge $B^{\omega_{1}, \pm \omega_{2}}$, or

in a cone about $L$ with a tube about $L$ excised,

$\hat{u}_{i d}$ is supported in a cone away from the above directions, i.e., in $G$.

Notice that $u_{i b}$ is conormal with respect to the plane perpendicular to the line $L$.

$$
\begin{aligned}
u_{1} u_{2}= & u_{1 a} u_{2 a}+u_{1 a} u_{2 b}+u_{1 a} u_{2 c}+u_{1 a} u_{2 d} \\
& +u_{1 b} u_{2 a}+u_{1 b} u_{2 b}+u_{1 b} u_{2 c}+u_{1 b} u_{2 d} \\
& +u_{1 c} u_{2 a}+u_{1 c} u_{2 b}+u_{1 c} u_{2 c}+u_{1 c} u_{2 d} \\
& +u_{1 d} u_{2 a}+u_{1 d} u_{2 b}+u_{1 d} u_{2 c}+u_{1 d} u_{2 d} .
\end{aligned}
$$

Now, analyzing each piece separately, using Schauder's Lemma, Rauch's Lemma, Proposition 11.3, and Proposition 11.5, we arrive at the desired result. 
Proposition 12.3. Let $f$ be a polynomial, $s>(n+1) / 2$, and let $u \in H^{s}\{(t, x)$ : $0 \leq t \leq T\}$ satisfy

$$
\square u=f(u),\left.\quad u\right|_{t=0}=\left.v\right|_{t=0},\left.\quad u_{t}\right|_{t=0}=\left.v_{t}\right|_{t=0},
$$

where $v \in H^{s}, \square v=0, v=v_{+}+v_{-}+\tilde{u}, v_{+}$and $v_{-}$are conormal with respect to a plane $\left(\Pi W F\left(v_{+}\right) \subset\{(\tau, \xi): \tau>0\}, \Pi W F\left(v_{-}\right) \subset\{(\tau, \xi): \tau<0\}\right)$, and $\tilde{u}$ has $\Pi W F(\tilde{u})$ in a single direction, away from $\Pi W F\left(v_{+}\right)$and $\Pi W F\left(v_{-}\right)$. Then $u \in H^{s, s, 2 s+1 / 2-\varepsilon, \infty}$.

Proof. We begin with $u \in H^{s, s, s, s}$, so $f(u) \in H^{s, s, s, s}$ by Schauder's Lemma. $E(f(u)) \in H^{s+1, s+1, s+1, s+1}$, since the forward fundamental solution $E$ for acts appropriately on these spaces. Now, $v \in H^{s, s, \infty, \infty}$. Since $u=v+$ $E(f(u)), u \in H^{s, s, s+1, s+1}$. Now assume that $u \in H^{s, s, r, g}, s \leq r \leq g$. Then $f(u) \in H^{s, s, r, g}$ by Lemma 12.2, as long as $r<2 s-1 / 2$. So $E(f(u)) \in$ $H^{s+1, s+1, r+1, g+1}$. Therefore, $u \in H^{s, s, r+1, g+1}$.

Continuing, by induction, we obtain $u \in H^{s, s, 2 s-1 / 2-\varepsilon, 2 s-1 / 2-\varepsilon}$. Then, by induction, assume $u \in H^{s, s, 2 s-1 / 2-\varepsilon, g}, g \geq 2 s-1 / 2-\varepsilon$. Then $f(u) \in$ $H^{s, s, 2 s-1 / 2-\varepsilon, g}$, and $E(f(u)) \in H^{s+1, s+1,2 s+1 / 2-\varepsilon, g+1}$, so $u \in H^{s, s, 2 s+1 / 2-\varepsilon, g+1}$. Continuing, we obtain $u \in H^{s, s, 2 s-1 / 2-\varepsilon, \infty}$. Finally, $f(u) \in H^{s, s, 2 s-1 / 2-\varepsilon, \infty}$, and $E(f(u)) \in H^{s+1, s+1,2 s+1 / 2-\varepsilon, \infty}$, so $u \in H^{s, s, 2 s+1 / 2-\varepsilon, \infty}$.

The microlocal regularity at $\{(t, x)=(0,0)\}$ again yields microlocal regularity along the corresponding null bicharacteristics, by Hörmander's theorem.

Corollary 12.4. Let $u, v, f$ be as above. Then $W F(u) \subset W F\left(v_{+}\right) \cup W F\left(v_{-}\right)$ $\cup W F(\tilde{u})$.

Thus, no additional singularities besides those present in the linear solution $v$ occur.

\section{INTERACTION OF TANGENTIAL WAVES}

Let $u$ solve $\square u=f(x, y, t, u)$. If $u$ has conormal singularities on three transversal characteristic hypersurfaces, then new singularities can arise on the light cone over the point of triple intersection. This was shown in Rauch-Reed [15] as well as Beals [2]. See Figure 12.

A natural question is, what happens if, at a later time, another characteristic hypersurface carrying conormal singularities intersects the line (or curve) where the light cone and an original hypersurface intersect tangentially? Therefore, we consider the problem of a solution $u$ with conormal singularities in the past on two tangential and one transversal hypersurface.

Note that in Melrose-Ritter [10], the presence of new singularities on the light cone arising out of the triple interaction with two tangent characteristic surfaces is necessary for the predicted dense singularities arising from a finite number of initial singularities conormal at a point. Melrose-Ritter also predicted that the extra singularities have higher Sobolev regularity than the initial singularities. In a related problem, Sá Baretto [17] studied the case of a solution conormal in the past to a hypersurface with a swallowtail singularity (a more complicated geometry than that considered here). He showed that the solution is conormal 


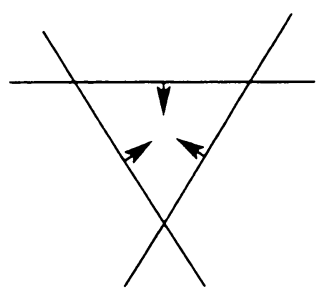

$t<0$

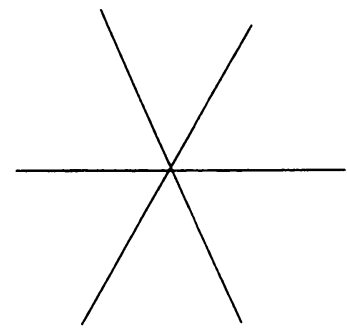

$t=0$

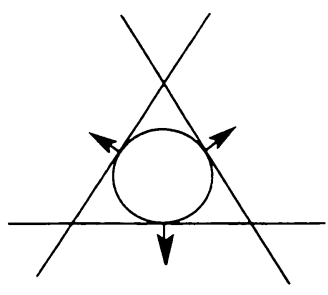

$t>0$

FIGURE 12. Nonlinear interaction of three planes

in the future with respect to the original hypersurface and the light cone arising out of intersection of the cusps.

It is convenient to consider an operator of the following form: $\tilde{\square}=\partial_{y} \partial_{z}-$ $\partial_{x}^{2}+y \partial_{y}^{2}-(m-1) \partial_{y}$ and $t=y+z$. The characteristic hypersurfaces to be considered are $y=0$ and $y=x^{2} / 4$, which intersect tangentially, and $z=0$, which is transversal to the other two. Here the time variable is $t=y+z$, and $\tilde{\square}$ is strictly hyperbolic with respect to $t$ on a neighborhood of 0 . (At the origin, the principal term in $\square=\partial_{y} \partial_{z}-\partial_{x}^{2}$ is the usual D'Alembertian, after a linear change of coordinates.) As is easily verified, the surface of the light cone over 0 for $\tilde{\square}$ is given by $\left\{(x, y, z): y\left(e^{z}-1\right)=\left(x^{2} / 4\right) e^{z}\right\}$.

The three waves to be considered are conormal to the following surfaces:

$v_{1}=z_{+}^{m}$ is conormal to $z=0$.

$v_{2}=y_{+}^{m}$ is conormal to $y=0$.

$v_{3}=\left(\frac{x^{2}}{4}-y\right)_{+}^{m-1 / 2}$ is conormal to $y=x^{2} / 4$.

Here the notation $x_{+}$means $x H(x)$, where $H(x)$ is the Heaviside function. Notice that, for $m \geq 0$ an integer and $\tilde{\square}$ (for the corresponding $m$ ) as above, $\tilde{\square} v_{i}=0, i=1,2,3$.

The following spaces will be useful in analyzing the regularity of the solution $u$ to $\tilde{\square} u=\beta u^{3}, \beta$ a $C_{0}^{\infty}$ function supported near 0 .

\section{Definition 13.1.}

$$
\begin{array}{r}
H_{k_{1}, k_{2}, k_{3}}^{s}\left(\mathbf{R}^{3}\right)=\left\{u \in L_{\mathrm{loc}}^{2}\left(\mathbf{R}^{3}\right) \cap L_{\mathrm{loc}}^{\infty}\left(\mathbf{R}^{3}\right): \partial_{z}^{c_{1}} \partial_{x}^{c_{2}}\left(2 \partial_{x}+x \partial_{y}\right)^{c_{3}} u \in H_{\mathrm{loc}}^{s}\left(\mathbf{R}^{3}\right)\right. \\
\left.\forall c_{i} \leq k_{i}, i=1,2,3\right\} .
\end{array}
$$

Similarly,

$$
H_{k_{1}, k_{2}, k_{3}}^{s_{-}}=\bigcap_{\varepsilon>0} H_{k_{1}, k_{2}, k_{3}}^{s-\varepsilon}
$$

Notice that each of the vector fields $\partial_{z}, \partial_{x}$, and $2 \partial_{x}+x \partial_{y}$ is tangent to two of the hypersurfaces under consideration. Moreover, away from $\{x=0\}$, these vector fields span $T\left(\mathbf{R}^{3}\right)$. Also notice that $v_{1} \in H_{0, \infty, \infty}^{m+1 / 2-}\left(\mathbf{R}^{3}\right), v_{2} \in$ $H_{\infty, \infty, 0}^{m+1 / 2-}\left(\mathbf{R}^{3}\right)$, and $v_{3} \in H_{\infty, 0, \infty}^{m-}\left(\mathbf{R}^{3}\right)$. 
Theorem 13.2. If we consider the system of equations:

$$
\begin{aligned}
& \tilde{\square}\left(\begin{array}{l}
u_{1} \\
u_{2} \\
u_{3} \\
u_{4}
\end{array}\right)=\left(\begin{array}{c}
0 \\
0 \\
0 \\
\beta u_{1} u_{2} u_{3}
\end{array}\right), \\
& \left(\begin{array}{l}
u_{1} \\
u_{2} \\
u_{3} \\
u_{4}
\end{array}\right)_{\{t<0\}}=\left(\begin{array}{c}
v_{1} \\
v_{2} \\
v_{3} \\
0
\end{array}\right)_{\{t<0\}},
\end{aligned}
$$

then $u_{1}=v_{1}, u_{2}=v_{2}, u_{3}=v_{3}$, and $v_{4}=E \beta\left(v_{1} v_{2} v_{3}\right)$ solves the system, and $u_{4}$ contains singularities on the surface of the light cone over the origin which are not present in the solution of the corresponding linear problem. In particular, let $K$ be the surface of the light cone minus the original hypersurfaces:

$$
K=\left\{(x, y, z) \mid y\left(e^{z}-1\right)=\frac{x^{2}}{4} e^{z}, y \neq 0, z \neq 0, y \neq \frac{x^{2}}{4}\right\} .
$$

Then for $\Omega$ a sufficiently small neighborhood of a point in $K$,

$$
E \beta\left(6 z_{+}^{m} y_{+}^{m}\left(\frac{x^{2}}{4}-y\right)_{+}^{m-1 / 2}\right) \notin H^{5 m+3}(\Omega) .
$$

The following two sections contain some preliminary results followed by the proof of the theorem.

\section{AuXILIARY RESUlts}

Several regularity estimates will be useful in the analysis of the solution to the system of equations.

The first lemma allows us to differentiate $u_{4}$ using any appropriate derivatives, and then add the total number of derivatives in determining the microlocal Sobolev regularity on the light cone away from the original singular surfaces.

Lemma 14.1. Assume $w \in H_{a, b, c}^{s}(\Omega)$ for $\Omega$ an open neighborhood of the surface of the light cone over the origin, away from the original hypersurfaces $y=0, z=$ $0, y=x^{2} / 4$. Then, microlocally near the conormal bundle to $K=\{(x, y, z)$ : $\left.y\left(e^{z}-1\right)=\frac{x^{2}}{4} e^{z}, y \neq 0, z \neq 0, y \neq x^{2} / 4\right\}, w \in H_{m l}^{s+a+b+c}\left(N^{*} K\right)$.

Proof. On $K, x \neq 0$, so $\left\{\partial_{z}, \partial_{x}, \partial_{x}+(x / 2) \partial_{y}\right\}$ is equivalent to $\left\{\partial_{z}, \partial_{x}, \partial_{y}\right\}$. Thus $\langle(\xi, \eta, \xi)\rangle^{s} \zeta^{a} \xi^{b} \eta^{c} \hat{w} \in L^{2}$. The normal direction to $y\left(e^{z}-1\right)=\frac{x^{2}}{4} e^{z}$ is $\left(-x / 2 e^{z}, e^{z}-1,\left(y-x^{2} / 4\right) e^{z}\right)$, and on $K, \xi \neq 0, \eta \neq 0, \zeta \neq 0$ since $x \neq 0, z \neq 0, y \neq x^{2} / 4$. So microlocally on $N^{*} K,|(\xi, \eta, \zeta)| \approx|\xi| \approx|\eta| \approx|\zeta|$. Therefore, on this set

$$
\langle(\xi, \eta, \zeta)\rangle^{s}|\xi|^{b}|\eta|^{c}|\zeta|^{a} \hat{w} \approx\langle(\xi, \eta, \zeta)\rangle^{s+a+b+c} \hat{w} \in L^{2}\left(\mathbf{R}^{3}\right) .
$$

Next, we need to know how the forward fundamental solution $E$ interacts with the defined spaces $H_{k_{1}, k_{2}, k_{3}}^{s}$. As with the usual Sobolev spaces, the regularity is improved by one. 
Lemma 14.2. Let $E$ be the forward fundamental solution for $\tilde{\square}=\partial_{z} \partial_{y}-\partial_{x}^{2}+$ $y \partial_{y}^{2}-(m-1) \partial_{y}$ and let $\beta \in C_{0}^{\infty}\left(\mathbf{R}^{3}\right)$ have support in $t \geq c$ where $t=y+z$ is a time variable. Then $E \beta w \in H_{k_{1}, k_{2}, k_{3}}^{s+1}$ for $w \in H_{k_{1}, k_{2}, k_{3}}^{s}$.

Proof. Let $\tilde{\square} u=\beta w, u=0$ for $t<c$, and let

$$
N=\left(\partial_{z}\right)^{k_{1}}\left(\partial_{x}\right)^{k_{2}}\left(2 \partial_{x}+x \partial_{y}\right)^{k_{3}} .
$$

Then

$$
\tilde{\square} N u-[\tilde{\square}, N] u=N \beta w .
$$

Note that $\partial_{x}$ and $\partial_{z}$ commute with $\tilde{\square}$, while

$$
\left[\tilde{\square}, 2 \partial_{x}+x \partial_{y}\right]=-\partial_{y}\left(2 \partial_{x}+x \partial_{y}\right) \text {. }
$$

It follows that

$$
\left[\tilde{\square},\left(2 \partial_{x}+x \partial_{y}\right)^{k_{3}}\right]=-k_{3} \partial_{y}\left(2 \partial_{x}+x \partial_{y}\right)^{k_{3}}
$$

and therefore

$$
[\tilde{\square}, N]=-k_{3} \partial_{y} N
$$

Consequently

$$
\left(\tilde{\square}+k_{3} \partial_{y}\right) N u=N \beta w \in H^{s}, \quad \text { and } \quad N u \equiv 0 \text { for } t<c .
$$

So $N u \in H^{s+1}$ by the usual energy estimate for the wave equation, and $u \in$ $H_{k_{1}, k_{2}, k_{3}}^{s+1}$. But, by uniqueness of the solution to the problem, $u \equiv E \beta w$.

The following lemma of Gagliardo-Nirenberg type is needed to show that $H_{k_{1}, k_{2}, k_{3}}^{s}$ is an algebra. See for example [4].

Lemma 14.3. Let $0 \leq s^{\prime} \leq s$ and suppose that $w \in L^{\infty}\left(\mathbf{R}^{3}\right) \cap H^{s}\left(\mathbf{R}^{3}\right)$. Then for $|\alpha|=s$, it follows that $D^{\alpha} w \in L^{2 s^{\prime} s^{\prime}}\left(\mathbf{R}^{3}\right)$ and

$$
\left\|D^{\alpha} w\right\|_{L^{2 s / s^{\prime}}} \leq C_{s, s^{\prime}, n}\left(\|w\|_{L^{\infty}}\right)^{1-s^{\prime} / s}\left(\|w\|_{H^{s}}\right)^{s^{\prime} / s} .
$$

Lemma 14.4. Suppose $w \in H_{k_{1}, k_{2}, k_{3}}^{s}\left(\mathbf{R}^{3}\right)$ for $s \geq 0$. If $f \in C^{\infty}(\mathbf{R})$, then $f(w)$ $\in H_{k_{1}, k_{2}, k_{3}}^{s}\left(\mathbf{R}^{3}\right)$. The same holds with $H_{k_{1}, k_{2}, k_{3}}^{s-}\left(\mathbf{R}^{3}\right)$ in place of $H_{k_{1}, k_{2}, k_{3}}^{s}\left(\mathbf{R}^{3}\right)$.

Proof. We need to show that $\partial_{z}^{k_{1}} \partial_{x}^{k_{2}}\left(2 \partial_{x}+x \partial_{y}\right)^{k_{3}} f(w) \in H^{s}\left(\mathbf{R}^{3}\right)$, i.e.,

$$
D^{\alpha} \partial_{z}^{k_{1}^{\prime}} \partial_{x}^{k_{2}^{\prime}}\left(2 \partial_{x}+x \partial_{y}\right)^{k_{3}^{\prime}} f(w) \in L^{2}
$$

where $k_{i}^{\prime} \leq k_{i}, i=1,2,3,|\alpha|=s$, and $D^{\alpha}$ stands for any derivative of order $s$. By the chain rule and Leibniz formula, $D^{\alpha} \partial_{z}^{k_{1}^{\prime}} \partial_{x}^{k_{2}^{\prime}}\left(2 \partial_{x}+x \partial_{y}\right)^{k_{3}^{\prime}} f(w)$ may be written as a sum of terms of the form

$$
g(w) D^{\alpha_{1}} \partial_{z}^{k_{11}} \partial_{x}^{k_{21}}\left(2 \partial_{x}+x \partial_{y}\right)^{k_{31}}(w) \cdots D^{\alpha_{m}} \partial_{z}^{k_{1 m}} \partial_{x}^{k_{2 m}}\left(2 \partial_{x}+x \partial_{y}\right)^{k_{3 m}}(w)
$$

where $\left|\alpha_{1}\right|+\cdots+\left|\alpha_{m}\right|=|\alpha|,\left|k_{i 1}\right|+\cdots+\left|k_{i m}\right|=k_{i}^{\prime}, i=1,2,3$. First note that $\partial_{z}^{k_{1 j}} \partial_{x}^{k_{2 j}}\left(2 \partial_{x}+x \partial_{y}\right)^{k_{3 j}}(w)=u_{j} \in H^{s}$, since $w \in H_{k_{1}, k_{2}, k_{3}}^{s}\left(\mathbf{R}^{3}\right)$. So we can apply Lemma 14.3 to each factor $D^{\alpha_{j}} u_{j}$ and conclude that

$$
D^{\alpha_{j}} u_{j}=f_{j} \in L^{2 s /\left|\alpha_{j}\right|} \text {. }
$$


Therefore (1) has the form $g_{\alpha}(w) f_{1} \cdots f_{m}$ with $g_{\alpha}(w) \in L_{\text {loc }}^{\infty}$ and $f_{j} \in L^{2 s /\left|\alpha_{j}\right|}$. Consequently

$$
\begin{aligned}
& \left\|g(w) D^{\alpha_{1}} \partial_{z}^{k_{11}} \partial_{x}^{k_{21}}\left(2 \partial_{x}+x \partial_{y}\right)^{k_{31}}(w) \cdots D^{\alpha_{m}} \partial_{z}^{k_{1 m}} \partial_{x}^{k_{2 m}}\left(2 \partial_{x}+x \partial_{y}\right)^{k_{3 m}}(w)\right\|_{L^{2}} \\
& =\left(\int\left(g(w) f_{1} \cdots f_{m}\right)^{2}\right)^{1 / 2} \leq\|g(w)\|_{L^{\infty}}\left(\int f_{1}^{2} \cdots f_{m}^{2}\right)^{1 / 2} \\
& \quad \leq\|g(w)\|_{L^{\infty}}\left[\left(\int f^{2 s /\left|\alpha_{1}\right|}\right)^{\left|\alpha_{1}\right| / s} \cdots\left(\int f^{2 s /\left|\alpha_{m}\right|}\right)^{\left|\alpha_{m}\right| / s}\right]^{1 / 2}
\end{aligned}
$$

since $\frac{\left|\alpha_{1}\right|}{s}+\cdots+\frac{\left|\alpha_{m}\right|}{s}=1$, using Hölder's Inequality. The last expression is equal to

$$
\|g(w)\|_{L^{\infty}}\left\|f_{1}\right\|_{L^{2 s /\left|\alpha_{1}\right|}} \cdots\left\|f_{m}\right\|_{L^{2 s / \alpha_{m} \mid}}
$$

The proof for $H_{k_{1}, k_{2}, k_{3}}^{s-}\left(\mathbf{R}^{3}\right)$ is similar.

\section{Proof OF TheOREM 13.2}

We need to show that there are singularities on the set $K$ consisting of the part of the surface of the light cone over 0 away from the original hypersurfaces, and determine the regularity of these singularities. In what follows, $c$ represents some constant, which can be different each time it occurs. The proof is written for $m$ even. If $m$ is odd, the proof is similar, except that the formulas for the exponents of $x$ and $\left(\frac{x^{2}}{4}-y\right)$ are slightly different.

$$
\begin{aligned}
\partial_{x}^{3 m+2}\left(2 \partial_{x}+x \partial_{y}\right)^{m} y_{+}^{m}\left(\frac{x^{2}}{4}-y\right)_{+}^{m-1 / 2} & \\
= & \partial_{x}^{3 m+2}\left(2 \partial_{x}+x \partial_{y}\right)^{m-1} c x y_{+}^{m-1}\left(\frac{x^{2}}{4}-y\right)_{+}^{(2 m-1) / 2} \\
= & \partial_{x}^{3 m+2}\left(2 \partial_{x}+x \partial_{y}\right)^{m-2}\left[c x^{2} y_{+}^{m-2}\left(\frac{x^{2}}{4}-y\right)_{+}^{(2 m-1) / 2}\right. \\
& \left.+c y_{+}^{m-1}\left(\frac{x^{2}}{4}-y\right)_{+}^{(2 m-1) / 2}\right] \\
= & \left.\partial_{x}^{3 m+2}\left(2 \partial_{x}+x \partial_{y}\right)^{m-2}\left[c x^{2} y_{+}^{m-2}\left(\frac{x^{2}}{4}-y\right)_{+}^{(2 m-1) / 2}\right)_{+}^{(2 m-2}\left(\frac{x^{2}}{4}-y\right)_{+}^{(2 m+1) / 2}\right] \\
& \left(\text { using } y=-\left(\frac{x^{2}}{4}-y\right)+\frac{x^{2}}{4}\right) .
\end{aligned}
$$


By induction, we obtain an expression of the form

$$
\begin{array}{r}
\partial_{x}^{3 m+2}\left(2 \partial_{x}+x \partial_{y}\right)\left[c x^{m-1} y+\left(\frac{x^{2}}{4}-y\right)_{+}^{(2 m-1) / 2}+c x^{m-3} y+\left(\frac{x^{2}}{4}-y\right)_{+}^{(2 m+1) / 2}\right. \\
\left.+\cdots+c x^{1} y_{+}\left(\frac{x^{2}}{4}-y\right)_{+}^{(3 m-3) / 2}\right] \\
=\partial_{x}^{3 m+2}\left(2 \partial_{x}+x \partial_{y}\right)\left[c x^{m+1} H(y)\left(\frac{x^{2}}{4}-y\right)_{+}^{(2 m-1) / 2}\right. \\
+c x^{m-1} H(y)\left(\frac{x^{2}}{4}-y\right)_{+}^{(2 m+1) / 2} \\
\left.+\cdots+c x^{1} H(y)\left(\frac{x^{2}}{4}-y\right)_{+}^{(3 m-1) / 2}\right] \\
=\partial_{x}^{3 m+2}\left[c x^{m+2} \delta(y)\left(\frac{x^{2}}{4}-y\right)_{+}^{(2 m-1) / 2}+c x^{m} \delta(y)\left(\frac{x^{2}}{4}-y\right)_{+}^{(2 m+1) / 2}\right. \\
\left.+\cdots+c x^{1} \delta(y)\left(\frac{x^{2}}{4}-y\right)_{+}^{(3 m-1) / 2}\right] \\
=\partial_{x}^{3 m+2}\left[c x^{m+2} \delta(y)|x|^{(2 m-1)}+c x^{m} \delta(y)|x|^{2 m+1}+\cdots+c x^{1} \delta(y)|x|^{3 m-1}\right] \\
=c \delta_{0}(x, y) .
\end{array}
$$

Therefore, $\partial_{z}^{m+1} \partial_{x}^{3 m+2}\left(2 \partial_{x}+x \partial_{y}\right)^{m} z_{+}^{m} y_{+}^{m}\left(\frac{x^{2}}{4}-y\right)_{+}^{m-1 / 2}=c \delta_{0}(x, y, z)$.

Now, $E \partial_{z}^{m+1} \partial_{x}^{3 m+2}\left(2 \partial_{x}+x \partial_{y}\right)^{m} z_{+}^{m} y_{+}^{m}\left(\frac{x^{2}}{4}-y\right)_{+}^{m-1 / 2}=F$, the fundamental solution for $\tilde{\square}$. To analyze the regularity of $E z_{+}^{m} y_{+}^{m}\left(\frac{x^{2}}{4}-y\right)_{+}^{m-1 / 2}$, we will use the above analysis, and the fact that $\left[\partial_{z}^{m+1} \partial_{x}^{3 m+2}\left(2 \partial_{x}+x \partial_{y}\right)^{m}\right.$, $\left.\tilde{\square}\right]=c \partial_{y}$, as shown in Lemma 14.2. Let $N=\partial_{z}^{m+1} \partial_{x}^{3 m+2}\left(2 \partial_{x}+x \partial_{y}\right)^{m}$, and $w=$ $z_{+}^{m} y_{+}^{m}\left(\frac{x^{2}}{4}-y\right)_{+}^{m-1 / 2}$. As is well known, $F \in H_{\text {loc }}^{-\varepsilon}(\Omega)$ for any $\varepsilon>0$, but $F \notin H_{\mathrm{loc}}^{0}(\Omega)$, if $\Omega \subset \mathbf{R}^{3}$ is any open set intersecting the surface of the light cone over the origin. (In the coordinates $t=x+y, s=x-y$, so that the principal part of $\tilde{\square}$ at the origin is $\partial_{t}^{2}-\left(\partial_{s}^{2}+\partial_{2}^{2}\right), F$ is essentially $\frac{c}{\sqrt{t^{2}-\left(s^{2}+z^{2}\right)}} \chi_{s^{2}+z^{2}-t^{2}}$.) We need to show that $N E w \notin H^{0}(\Omega)$, if $\Omega$ is a neighborhood of a point in $K$. Let $\tilde{\square} U=\beta w$, so that $N \tilde{\square} U=N \beta w$. Then

$$
\tilde{\square} N U-[\tilde{\square}, N] U=N \beta w,
$$

so

$$
\left(\tilde{\square}+c \partial_{y}\right) N U=N \beta w=c \delta_{0}(x, y, z)
$$

modulo more regular terms resulting from differentiating $\beta$. Then

$$
N U=F^{\prime}+G,
$$

with $F^{\prime}$ the fundamental solution for $\tilde{\square}+c \partial_{y}$ and $G$ smoother than $F^{\prime}$. Therefore $N U \in H_{\text {loc }}^{-\varepsilon}\left(\mathbf{R}^{3}\right), N U \notin H^{0}(\Omega)$. Since $U=E \beta w$, it follows that $E \beta w \in H_{m+1,3 m+2, m}^{0-}\left(\mathbf{R}^{3}\right), E \beta w \notin H_{m+1,3 m+2, m}^{0}\left(\mathbf{R}^{3}\right)$. In particular, since the singularities of $w$ locally on $K$ are microlocally concentrated on $N^{*} K$, as before we have $E \beta w \in H_{\mathrm{loc}}^{5 m+3-\varepsilon}(\Omega)$, but $E \beta w \notin H_{\mathrm{loc}}^{5 m+3}(\Omega)$. 


\section{REFERENCES}

1. M. Beals, Self-spreading and strength of singularities for solutions to semilinear wave equations, Ann. of Math. 118 (1983), 187-214.

2. _ Singularities of conormal radially smooth solutions to nonlinear wave equations, Comm. Partial Differential Equations 13 (1988), 1355-1382.

3. _ Vector fields associated with the nonlinear interaction of progressing waves, Indiana Univ. Math. J. 37 (1988), 637-666.

4. _ Propagation and interaction of singularities in nonlinear hyperbolic problems, Birkhäuser, Boston, 1989.

5. J. M. Bony, Second microlocalization and propagation of singularities for semilinear hyperbolic equations, Taniguchi Sympos., Katata, 1984, pp. 11-49.

6. __ Interaction des singularités pour les équations de Klein-Gordon non linéaires, Sém. Goulaouic-Meyer-Schwartz, Exp. no. 10, 1983-84.

7. J. Chazarain and A. Piriou, Introduction to the theory of linear partial differential equations, North-Holland, Amsterdam, 1982.

8. L. Hörmander, The analysis of linear partial differential operators. I, Springer-Verlag, Berlin, 1983.

9. L. Holt, Singularities produced in conormal wave interactions, Ph.D. thesis, Rutgers Univ., New Brunswick, N.J., 1991.

10. R. Melrose and N. Ritter, Interaction of progressing waves for semilinear wave equations, Ark. Mat. 25 (1987), 91-114.

11. L. Nirenberg, Lectures on linear partial differential equations, C.B.M.S. Regional Conf. Ser. in Math., no. 17, Amer. Math. Soc., Providence, R.I., 1973.

12. J. Rauch, Singularities of solutions to semilinear wave equations, J. Math. Pures Appl. 58 (1979), 299-308.

13. J. Rauch and M. Reed, Propagation of singularities for semilinear hyperbolic equations in one space variable, Ann. of Math. 111 (1980), 531-552.

14. __ Nonlinear microlocal analysis of semilinear hyperbolic systems in one space dimension, Duke Math. J. 49 (1982), 379-475.

15. __ Singularities produced by the nonlinear interaction of three progressing waves, Comm. Partial Differential Equations 7 (1982), 1117-1133.

16. A. Sá Barreto, Second microlocal ellipticity and propagation of conormality for semilinear wave equations, J. Funct. Anal. 102 (1991), 47-71.

17. __ Evolution of semilinear waves with swallowtail singularities, preprint.

Department of Mathematics, California State University, San Marcos, 333 S. Twin Oaks Valley Rd., San Marcos, California 92096

E-mail address: linda_holtecsusm.edu 